\title{
LIPSCHITZ AND QUASICONFORMAL APPROXIMATION AND EXTENSION
}

\author{
P. TUKIA and J. VÄISÄLÄ
}

\section{Introduction}

D. Sullivan has recently given an important variation of the theory of R. D. Edwards and R. C. Kirby [EK] on the deformation of embeddings. The essential difference between these theories is that Sullivan replaces the $n$-torus $T^{n}$ by a more complicated closed manifold $Q^{n}$ and the natural covering map $e: R^{n} \rightarrow T^{n}$ by a covering map $e^{\prime}: B^{n} \rightarrow Q^{n}$ where $B^{n}$ is the open unit ball in $R^{n}$. Sullivan's theory is particularly useful in the categories of quasiconformal and lipschitz maps. For example, he proved in both categories the annulus theorem in all dimensions. Moreover, he established approximation theorems, which implied the existence of a lipschitz structure on every topological $n$-manifold without boundary for $n \neq 4$, and also the hauptvermutung for these manifolds in both categories. For $n \leqq 3$ these results can also be obtained by PL methods, see $\left[\mathrm{Tu}_{1}\right]$ and [Vä].

In this paper, we first develop Sullivan's theory for manifolds which may have boundary. For example, we prove in both categories the hauptvermutung for $n$-manifolds with boundary, $n \neq 4,5$. In the quasiconformal case our proof makes use of the extension of a quasiconformal map from $R^{n}$ to $R_{+}^{n+1}$, proved by the authors in $\left[\mathrm{TV}_{2}\right]$ with the aid of Sullivan's theory.

Since the presentation of $[\mathrm{Su}]$ is very sketchy, a large part of this article (most of Sections 2-4) is devoted to a fairly detailed exposition of Sullivan's theory. We take on faith the most difficult part, namely the existence of the Sullivan groups (defined in 2.9), and we assume that the reader is familiar with the basic ideas of [EK], but otherwise our presentation is reasonably self-contained.

We also keep track of the dilatations and the bilipschitz constants of the maps, and obtain quantitative versions of Sullivan's results. These are applied in Section 5 to give estimates for these constants in certain extension problems. For example, we obtain a dilatation estimate in the quasiconformal Schoenflies theorem, previously known only in a very special case. In Section 7 we consider the approximation of quasiconformal and certain more general homeomorphisms by homeomorphisms which satisfy a bilipschitz condition, either locally in the euclidean metric 
or globally in the quasihyperbolic metric. This metric and the associated notion of a solid homeomorphism are considered in detail in Section 6.

We thank Jouni Luukkainen for a careful reading of our manuscript and for several useful comments and corrections.

\section{Basic constructions}

2.1. Notation and terminology. The one-point compactification of the euclidean $n$-space $R^{n}$ is written as $\bar{R}^{n}=R^{n} \cup \infty$. If $m<n$, we identify $R^{m}$ with the subspace $R^{m} \times 0$ of $R^{n}=R^{m} \times R^{n-m}$. Let $(X, d)$ be a metric space. If $a \in X$ and $r>0$, we let $B_{d}(a, r)$ or $B(a, r)$ denote the open ball $\{x \in X: d(x, a)<r\}$. More generally, if $\emptyset \neq A \subset X$, we write $B(A, r)=\{x \in X: d(x, A)<r\}$ where $d(x, A)$ is the distance between $x$ and $A$. If $A$ and $B$ are nonempty subsets of $X$, we let $d(A, B)$ denote the distance between $A$ and $B$ and $d(A)$ the diameter of $A$. If $X=R^{n}$, we let $d$ denote the standard euclidean metric, and write

$$
\begin{gathered}
B^{n}(x, r)=B(x, r), \quad B^{n}(r)=B(0, r), \quad B^{n}=B(0,1), \\
S^{n-1}(x, r)=\partial B(x, r), \quad S^{n-1}(r)=S^{n-1}(0, r), \quad S^{n-1}=S^{n-1}(0,1) .
\end{gathered}
$$

The closed unit cube $[-1,1]^{n}$ of $R^{n}$ is denoted by $I^{n}$, and the open cube $(-r, r)^{n}$ by $I^{n}(r)$. However, we set $I=[0,1]$, although $I^{1}=[-1,1]$. The upper half space of $R^{n}$ is $R_{+}^{n}=\left\{x \in R^{n}: x_{n} \geqq 0\right\}$. If $A$ is any subset of $R^{n}$, we set $A_{+}=A \cap R_{+}^{n}$.

If $f$ and $g$ are maps into a metric space $(Y, d)$, defined in a set $A$, we write

$$
d(f, g ; A)=\sup \{d(f(x), g(x)): x \in A\} .
$$

Let $(X, d)$ and $\left(Y, d^{\prime}\right)$ be metric spaces. A map $f: X \rightarrow Y$ is $L$-lipschitz, $L \geqq 0$, if

$$
d^{\prime}(f(x), f(y)) \leqq L d(x, y)
$$

for all $x, y \in X$. If $L \geqq 1$ and if, in addition,

$$
d^{\prime}(f(x), f(y)) \geqq d(x, y) / L,
$$

$f$ is $L$-bilipschitz. If each point $x$ of $X$ has a neighborhood $U$ such that $f \mid U$ is lipschitz, $L$-lipschitz, bilipschitz or $L$-bilipschitz, $f$ is said to be locally lipschitz, locally $L$-lipschitz, locally bilipschitz or locally $L$-bilipschitz, respectively. We let LIP denote the category of metric spaces and locally lipschitz (=LIP) maps. A locally bilipschitz embedding or immersion is said to be a LIP embedding or a LIP immersion, respectively.

Let $A \subset \bar{R}^{n}$ be a set such that $A \subset \mathrm{cl}$ int $A$. If $n \geqq 2$, an embedding $f: A \rightarrow \bar{R}^{n}$ is said to be $K$-quasiconformal or $K$-QC if its restriction to each component of int $A$ is $K$-QC in the sense of [V $\left.\ddot{a}_{1}\right]$. If $n=1, f$ is said to be $K$-quasiconformal if 
its restriction to each component of int $A$ is $K$-quasisymmetric [ $\mathrm{LeV}_{2}$, II.7.1]. We allow the possibility that $f$ is sense-reversing. The concept "locally quasiconformal" (LQC) is defined in the obvious way.

We can define LQC manifolds and LIP manifolds in the well-known manner using atlases (see also [LuV, §3] and [LT, 4.6]). Observe that every LIP manifold is an LQC manifold.

If $A \subset B$, we let id denote the inclusion map $A \rightarrow B$.

If $X$ and $Y$ are metric spaces, we shall consider the space $C(X, Y)$ of all continuous maps $f: X \rightarrow Y$ endowed with the compact-open topology. We let $H(X) \subset$ $C(X, X)$ denote the group of all homeomorphisms $f: X \rightarrow X$.

If $A$ is a subset of a topological space $X$, we let "near $A$ " mean "in a neighborhood of $A$ ".

If $A \subset R^{n}$, if $f: A \rightarrow R^{p}$ is a map and if $x_{0} \in A$ is an accumulation point of $A$, we set

$$
L\left(x_{0}, f\right)=\limsup _{x \rightarrow x_{0}} \frac{\left|f(x)-f\left(x_{0}\right)\right|}{\left|x-x_{0}\right|} .
$$

If $\alpha$ is a rectifiable path in $R^{n}, l(\alpha)$ will denote its euclidean length.

The 1-dimensional case needs often a special treatment, since the properties of 1-dimensional quasisymmetric maps are different from the properties of higherdimensional quasiconformal maps. On the other hand, it is usually easy to give a direct proof for the 1-dimensional case. To avoid technicalities, we make the following convention: The symbol $n$ is always an integer at least two, unless otherwise stated.

The following elementary lemma is well-known (see, for example, [Fe, p. 64]):

2.2. Lemma. Suppose that $A$ is a convex set in $R^{p}$ and that $f: A \rightarrow R^{m}$ is a map such that $L(x, f) \leqq L_{0}$ for every $x \in A$. Then $f$ is $L_{0}$-lipschitz.

2.3. Möbius transformations. We let $G M(m)$ denote the group of all Möbius transformations of $\bar{R}^{m}$. Thus $G M(m)$ is generated by similarity maps and inversions in spheres (in fact, by inversions alone). The image of a $p$-sphere under a Möbius transformation is always a $p$-sphere, if we regard a $p$-plane as a $p$-sphere through $\infty$.

We let $M \mathrm{Mob}_{m}$ denote the subgroup of $G M(m)$ consisting of maps $g$ such that $g B^{m}=B^{m}$. Then $\mathrm{Möb}_{m}$ is generated by inversions in all orthogonal spheres of $S^{m-1}$. The group of all sense-preserving maps in $\mathrm{Möb}_{m}$ is written as $\mathrm{Möb}_{m}^{+}$. There is a natural topology in $G M(m)$, which can be defined in several ways. It is the topology of pointwise convergence and also the compact-open topology. Moreover, the induced topology of $\mathrm{Möb}_{m}$ can be defined by the metric $d\left(f, g ; B^{m}\right)$.

2.4. Hyperbolic metric. Let $0 \leqq p \leqq m-1$, and let $T \subset \bar{R}^{m}$ be a $p$-plane (affine subspace). The hyperbolic metric $\sigma_{T}$ of $R^{m} \backslash T$ is defined by the element of length $d \sigma_{T}=|d x| / d(x, T)$. This means that if $a, b \in R^{m} \backslash T, \sigma_{T}(a, b)$ is the infimum of the 
line integrals

$$
\int_{\alpha} \frac{|d x|}{d(x, T)}
$$

taken over all rectifiable paths $\alpha$ joining $a$ and $b$ in $R^{m} \backslash T$. If $H$ is a half space whose boundary contains $T$, and if $a, b \in H$, it is sufficient to consider paths $\alpha$ in $H$. If $p=m-1, \sigma_{T}(a, b)$ is defined only if $a$ and $b$ belong to the same component of $R^{m} \backslash T$. The metric $\sigma_{T}$ is invariant under the Möbius transformations $g$ which respect $T$, that is, $g T=T$. Indeed, this is easy to verify if $g$ is a similarity or if $g(x)=$ $x /|x|^{2}$, and the general case follows from these.

Let $S \subset R^{m}$ be a $p$-sphere of radius $r$. The hyperbolic metric $\sigma_{S}$ in $\bar{R}^{m} \backslash S$ is defined as follows: Choose $h \in G M(m)$ such that $h S$ is a $p$-plane $T$. Then

$$
\sigma_{S}(a, b)=\sigma_{T}(h(a), h(b)) \text {. }
$$

If $p=m-1, \sigma_{S}(a, b)$ is again defined only if $a$ and $b$ belong to the same component of $\bar{R}^{m} \backslash S$. The metric $\sigma_{S}$ is independent of the choice of $h$, and it is invariant under the Möbius transformations which respect $S$. It can also be defined explicitly by the element of length

where

$$
d \sigma_{S}(x)=\frac{2 r|d x|}{d(x, S) D(x, S)},
$$

$$
D(x, S)=\max \{|x-y|: y \in S\} .
$$

In particular, if $S=S^{m-1}$, the hyperbolic metric of $B^{m}$ is defined by

$$
d \sigma_{S}(x)=\frac{2|d x|}{1-|x|^{2}} .
$$

The formula (2.6) follows by direct computation. However, we shall only need the fact that $d \sigma_{S}(x) d(x, S) /|d x|$ is bounded away from 0 and $\infty$ near $S$. We shall often omit the subscript $S$ from $\sigma_{S}$ if there is no danger of misunderstanding.

If $p=m-1$, a hyperbolic ball $B_{\sigma}(x, r)$ is always a euclidean ball or a half space or the exterior of a euclidean ball.

2.7. Möbius coordinates. Let $m$ and $k$ be positive integers, and set $n=m+k$. For every $z \in \bar{R}^{n} \backslash S^{m-1}$ we define the Möbius coordinates $\xi \in B^{m}$ and $\eta \in \bar{R}^{k}$ as follows: There is a unique orthogonal $k$-sphere $S_{1}^{k}$ of $S^{n-1}$ through $z$ which intersects $B^{m}$ orthogonally. (Again, $k$-planes are considered as $k$-spheres through $\infty$.) Then $\xi$ is the unique point of $S_{1}^{k} \cap B^{m}$. Furthermore, there is a unique $m$-sphere $S_{2}^{m}$ through $S^{m-1}$ and $z$. Let $C$ be the $z$-component of $S_{2}^{m} \backslash S^{m-1}$. Then $(0, \eta)$ is the unique point of $C \cap\left(0 \times R^{k}\right)$. Each point $z$ is uniquely determined by its Möbius coordinates, and we write $z=[\xi, \eta]$. All this is easy to see if $m=k=1$, and the general case follows by considering two-dimensional sections through $0, z$, and the orthogonal projection of $z$ in $R^{m}$. If $z \in S^{m-1}$, we set $\xi=z$, but $\eta$ is not defined. 
2.8. Lemma. Let $g \in \mathrm{Möb}_{m}$. Then the map $g^{*}$ defined by $g^{*}[\xi, \eta]=[g(\xi), \eta]$ is in $\mathrm{Möb}_{n}$.

Proof. If $g$ is a reflection in an orthogonal $(m-1)$-sphere of $S^{m-1}$, then $g^{*}$ is the reflection in the $(n-1)$-sphere with the same center and the same radius. Hence $g^{*} \in$ Möb $_{n}$. The general case follows from this.

2.9. Sullivan groups. Let $G$ be a subgroup of $\mathrm{Möb}_{m}^{+}$. We consider $G$ as a transformation group of $B^{m}$. Assume that $G$ has the following properties:

(1) $G$ is discrete.

(2) $G$ acts freely on $B^{m}$. This means that $g(x) \neq x$ whenever $x \in B^{m}, g \in G$ and $g \neq$ id.

Each orbit $G x, x \in B^{m}$, is discrete and closed in $B^{m}$. Let $Q$ be the orbit space $B^{m} / G$ with the quotient topology. The natural map $\pi_{0}: B^{m} \rightarrow Q$ is a covering map, and $Q$ is an $m$-manifold without boundary. Moreover, $\pi_{0}$ defines a DIFF (and hence LIP and LQC) structure on $Q$. The hyperbolic metric $\sigma_{Q}$ of $Q$ is defined as follows: If $p, q \in Q, \sigma_{Q}(p, q)$ is the hyperbolic distance of $\pi_{0}^{-1}(p)$ and $\pi_{0}^{-1}(q)$ as subsets of $B^{m}$. Then $\pi_{0}: B^{m} \rightarrow Q$ is locally a hyperbolic isometry. Indeed, set $r(x)=\sigma(x,(G x \backslash x)) / 4$. Then $\pi_{0}$ embeds $B_{\sigma}(x, r(x))$ isometrically onto

$$
B_{\sigma_{Q}}\left(\pi_{0}(x), r(x)\right)
$$

The LIP structure of $Q$ is also defined by the metric $\sigma_{Q}$. Each pair $p, q \in Q$ can be joined by a hyperbolic geodesic.

We say that $G$ is a Sullivan group if, in addition, it has the following two properties:

(3) $B^{m} / G=Q$ is compact.

(4) If $q \in Q$, there is a LIP immersion $\alpha: Q \backslash q \rightarrow R^{m}$.

The orbit space $Q$ will be called a Sullivan manifold. By a deep result of Sullivan [Su], a Sullivan group exists for every $m$. We fix for each positive integer $m$ a Sullivan group $G^{m}$ and the corresponding Sullivan manifold $Q^{m}=B^{m} / G^{m}$. The point $\pi_{0}(0) \in Q^{m}$ will be denoted by $q_{0}$.

For every $m$ we fix $r_{m} \in(0,1 / 2)$ such that $\pi_{0}: B^{m} \rightarrow Q^{m}$ is a hyperbolic isometry in a neighborhood of each closed hyperbolic ball of radius $r_{m}$. This is possible by the compactness of $Q^{m}$. Then, if $0<r \leqq r_{m}$ and $q \in Q^{m}$, the pre-image of $B_{\sigma_{Q}}(q, r)$ is a disjoint union of hyperbolic balls in $B^{m}$, and $\pi_{0}$ maps each of these isometrically onto $B_{\sigma_{o}}(q, r)$. We let $D^{m}$ denote the hyperbolic ball $B_{\sigma}\left(0, r_{m}\right) \subset B^{m}$ and $s_{m}=$ $\tanh \left(r_{m} / 2\right)$ the euclidean radius of $D^{m}$.

2.10. Some constructions. Let $m$ and $k$ be positive integers. Set $n=m+k$ and $A_{k}^{n}=\bar{B}^{n} \backslash S^{m-1}$. Then each $z \in A_{k}^{n}$ has Möbius coordinates $\xi \in B^{m}$ and $\eta \in \bar{B}^{k}$; see 2.7. We thus obtain a natural homeomorphism $u: A_{k}^{n} \rightarrow B^{m} \times \bar{B}^{k}$ defined by $u[\xi, \eta]=$ $(\xi, \eta)$. Setting $\pi=\pi_{0} \times$ id: $B^{m} \times \bar{B}^{k} \rightarrow Q_{m} \times \bar{B}^{k}$ and $e=\pi u$ we obtain the commuta- 
tive diagram

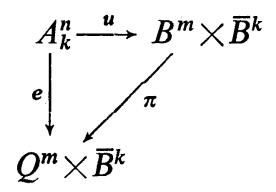

Here $\pi$ and $e$ are covering maps. Observe that $e(0)=\left(q_{0}, 0\right)$. The map $e$ plays an important role in the straightening of $k$-handles on an $n$-manifold. It corresponds to the natural covering map $R^{m} \times I^{k} \rightarrow T^{m} \times I^{k}$ of the Edwards-Kirby theory. For 0-handles we define $A_{0}^{n}=B^{n}$ and $e=\pi_{0}: B^{n} \rightarrow Q^{n}$. For $n$-handles we set $A_{n}^{n}=\bar{B}^{n}$ and $e=\mathrm{id}: \bar{B}^{n} \rightarrow \bar{B}^{n}$.

In $A_{k}^{n}$ we have the euclidean metric $d$ of $R^{n}$ and the hyperbolic metric $\sigma$ of $\bar{R}^{n} \backslash S^{m-1}$. In $B^{n} \times \bar{B}^{k}$ we use the metric $\varrho$ defined by

$$
\varrho\left(x, x^{\prime}\right)=\max \left(\sigma\left(x_{1}, x_{1}^{\prime}\right),\left|x_{2}-x_{2}^{\prime}\right|\right) .
$$

Replacing $\sigma$ by $\sigma_{Q}$ we obtain a metric on $Q^{m} \times \bar{B}^{k}$, also denoted by $\varrho$. Then $\pi$ is an isometry in each set of the form $B_{\sigma}\left(x, r_{m}\right) \times \bar{B}^{k}$.

2.11. Lemma. The homeomorphism $u:\left(A_{k}^{n}, \sigma\right) \rightarrow\left(B^{m} \times \bar{B}^{k}, \varrho\right)$ is bilipschitz.

Proof. Set $F=\bar{B}^{m}(1 / 2)$ and $E=u^{-1}\left[F \times \bar{B}^{k}\right]$. Since $u$ is a diffeomorphism, $u \mid E$ is $L$-bilipschitz for some $L$. Define the action of $\mathrm{Möb}_{m}$ on $B^{m} \times \bar{B}^{k}$ by $g\left(x_{1}, x_{2}\right)=$ $\left(g x_{1}, x_{2}\right)$. Then every $g: B^{m} \times \bar{B}^{k} \rightarrow B^{m} \times \bar{B}^{k}$ is a $\varrho$-isometry. Since $g u=u g$ for all $g \in \mathrm{Möb}_{m}, u \mid g E$ is $L$-bilipschitz for every $g \in \mathrm{Möb}_{m}$. Since the sets $g E$ cover $A_{k}^{n}$, it easily follows that $u$ is $L$-lipschitz and that $u^{-1}$ is $2 L$-lipschitz.

2.12. Lemma. Suppose that $0 \leqq k \leqq n-1$ and that $f: A_{k}^{n} \rightarrow A_{k}^{n}$ is a homeomorphism such that $f$ is locally L-bilipschitz in the hyperbolic metric $\sigma$ of $\bar{R}^{n} \backslash S^{m-1}$ and such that $\sigma(f, \mathrm{id}) \leqq M<\infty$. Then $f$ is $L_{1}$-bilipschitz in the euclidean metric of $R^{n}$ with $L_{1}=2 L e^{M}$.

Proof. It suffices to show that $f$ is $L_{1}$-lipschitz. Setting $\delta(x)=d\left(x, S^{m-1}\right)$ we obtain by (2.6)

$$
L(x, f) \leqq 2 L \delta(f(x)) / \delta(x)
$$

for all $x \in A$. By Lemma 2.2, it suffices to show that $\delta(f(x)) / \delta(x) \leqq e^{M}$. Let $x \in A_{k}^{n}$ and let $\alpha$ be a rectifiable path joining $x$ and $f(x)$ in $A_{k}^{n}$. Since $\delta: A_{k}^{n} \rightarrow R^{1}$ is 1-lipschitz, [Vä1, 5.3] implies

Hence

$$
\int_{\alpha} \frac{|d y|}{\delta(y)} \geqq \int_{\delta \circ \alpha} \frac{|d t|}{t} \geqq \log \frac{\delta(f(x))}{\delta(x) .} .
$$

$$
\frac{\delta(f(x))}{\delta(x)} \leqq e^{\sigma(f, \text { id })} \leqq e^{M} .
$$


2.13. Lifting of maps. Since $B^{m} \times \bar{B}^{k}$ is simply connected and $\pi: B^{m} \times \bar{B}^{k} \rightarrow$ $Q^{m} \times \bar{B}^{k}$ is a covering map, every (continuous) map $f: Q^{m} \times \bar{B}^{k} \rightarrow Q^{m} \times \bar{B}^{k}$ can be lifted to a map $\hat{f}: B^{m} \times \bar{B}^{k} \rightarrow B^{m} \times \bar{B}^{k}$ such that $\pi \hat{f}=f \pi$. The lifting $\hat{f}$ is unique as soon as we know $\hat{f}(0)$, which can be chosen arbitrarily in $\pi^{-1} f\left(q_{0}, 0\right)$.

The set

$$
\mathscr{P}_{0}=\left\{f \in H\left(Q^{m} \times \bar{B}^{k}\right): \varrho(f, \text { id })<r_{m} / 2\right\}
$$

is a neighborhood of id in $H\left(Q^{m} \times \bar{B}^{k}\right)$. In what follows, we shall only consider liftings of maps $f \in \mathscr{P}_{0}$. We let $\hat{f}$ denote the unique lifting of $f \in \mathscr{P}_{0}$ satisfying $\hat{f}(0) \in B_{\sigma}\left(0, r_{m} / 2\right) \times \bar{B}^{k}$. The map $\hat{f}$ is a self-homeomorphism of $B^{m} \times \bar{B}^{k}$. Indeed, if $f \in \mathscr{P}_{0}$, then also $f^{-1} \in \mathscr{P}_{0}$, and $\left(f^{-1}\right)^{\wedge}=(\hat{f})^{-1}$. The lifting $\hat{f}$ can also be directly defined as follows: For $x=\left(x_{1}, x_{2}\right) \in B^{m} \times \bar{B}^{k}$ let

$$
\pi_{x}: B_{\sigma}\left(x_{1}, r_{m}\right) \times \bar{B}^{k} \rightarrow B_{\sigma_{Q}}\left(\pi_{0}\left(x_{1}\right), r_{m}\right) \times \bar{B}^{k}
$$

be the isometry defined by $\pi$. Then $f(\pi(x)) \in \operatorname{im} \pi_{x}$, and we can define $\hat{f}(x)=$ $\pi_{x}^{-1} f \pi(x)$. From this description it is easy to see that $\varrho\left(\hat{f}_{1}, \hat{f}_{2}\right)=\varrho\left(f_{1}, f_{2}\right)$ for all $f_{1}, f_{2} \in \mathscr{P}_{0}$. In particular, $\varrho(\hat{f}$, id $)<r_{m} / 2$ for every $f \in \mathscr{P}_{0}$. Furthermore, if the action of $\mathrm{Möb}_{m}$ on $B^{m} \times \bar{B}^{k}$ is defined by $g\left(x_{1}, x_{2}\right)=\left(g x_{1}, x_{2}\right)$, then $g \hat{f}=\hat{f} g$ for every $f \in \mathscr{P}_{0}$ and $g \in G^{m}$.

We define the action of $\mathrm{Möb}_{m}$ on $A_{k}^{n}$ by $g[\xi, \eta]=[g(\xi), \eta]$. By Lemma 2.8 this action is conformal. For $f \in \mathscr{P}_{0}$ we set $f^{*}=u^{-1} \hat{f} u: A_{k}^{n} \rightarrow A_{k}^{n}$. Then $f^{*}$ is an $e$-lifting of $f$, that is, $e f^{*}=f e$. From the above considerations and from Lemma 2.11 we easily obtain the following properties of $f^{*}$ :

2.14. Lemma. The correspondence $f \mapsto f^{*}$ is a continuous map $\mathscr{P}_{0} \rightarrow H\left(A_{k}^{n}\right)$. If $f \in \mathscr{P}_{0}$ and $g \in G^{m}, g f^{*}=f^{*} g$. There is a constant $M_{n}$ depending only on $n$ such that $\sigma\left(f^{*}, \mathrm{id}\right) \leqq M_{n}$ for all $f \in \mathscr{P}_{0}$. Every $f^{*}$ can be extended by $\mathrm{id} \mid S^{m-1}$ to a homeomorphism $\bar{f}: \bar{B}^{n} \rightarrow \bar{B}^{n}$.

2.15. Local and global bilipschitz constants. It is usually much easier to show that a given embedding is locally $L$-bilipschitz than $L$-bilipschitz. For example, let $G$ be open in $R^{n}$, and let $f: G \rightarrow R^{n}$ be an embedding. Suppose that $G$ can be expressed as a locally finite union of sets $A_{j}$ such that $f \mid A_{j}$ is $L$-bilipschitz for every $j$. Then $f \mid \bar{A}_{j} \cap G$ is also $L$-bilipschitz, and the local convexity of $R^{n}$ implies that $f$ is locally $L$-bilipschitz. However, $f$ need not be bilipschitz even if $G=G_{1} \cup G_{2}$ with $f \mid G_{j}$ bilipschitz. However, we show that if we restrict the embeddings to a compact subset of $G$, the difference between $L$-bilipschitz and locally $L$-bilipschitz maps becomes fairly small. For this, we need the following result of F. John [Jo, Theorems II and III]:

2.16. Lemma. Suppose that $f: B^{n} \rightarrow R^{n}$ is a locally L-bilipschitz immersion with $f(0)=0$. Then

(1) $B^{n}(1 / L) \subset f B^{n} \subset B^{n}(L)$,

(2) $f \mid B^{n}\left(1 / L^{2}\right)$ is L-bilipschitz. 
2.17. Lemma. Let $G$ be open in $R^{n}$, and let $A$ be compact in $G$. Let $f: G \rightarrow R^{n}$ be a locally L-bilipschitz embedding. Then $(f \mid A)^{-1}$ is $L_{1}$-lipschitz with $L_{1}$ depending only on $G, A$ and $L$. If $G$ is connected, $f \mid A$ is $L_{1}$-bilipschitz.

Proof. We may assume that $G \neq R^{n}$. Set $r=d(A, \partial G)$, and let $x_{1}, x_{2} \in A$. If $\left|x_{1}-x_{2}\right| \leqq r / L^{2}$, Lemma 2.16 implies $\left|f\left(x_{1}\right)-f\left(x_{2}\right)\right| \geqq\left|x_{1}-x_{2}\right| / L$. Suppose that $\left|x_{1}-x_{2}\right| \geqq r / L^{2}$. Then the balls $B^{n}\left(x_{j}, r / 2 L^{2}\right)$ are disjoint. By Lemma 2.16, $B^{n}\left(f\left(x_{j}\right), r / 2 L^{3}\right) \subset f B^{n}\left(x_{j}, r / 2 L^{2}\right)$, which implies

$$
\left|f\left(x_{1}\right)-f\left(x_{2}\right)\right| \geqq r / L^{3} \geqq r\left|x_{1}-x_{2}\right| / L^{3} d(A) .
$$

Hence $(f \mid A)^{-1}$ is $L_{1}$-lipschitz with $L_{1}=\max \left(L, L^{3} d(A) / r\right)$.

Next assume that $G$ is connected. For $a, b \in G$ let $d_{1}(a, b)$ be the infimum of the lengths of all rectifiable paths $\alpha$ joining $a$ and $b$ in $G$. Then $d_{1}$ is a metric of $G$ defining its usual topology. Since $A$ is compact, $d_{1}(A)<\infty$. Suppose that $x, y \in A$. If $|x-y|<r$, the segment $x y$ lies in $G$, which implies $|f(x)-f(y)| \leqq L|x-y|$. Assume that $|x-y| \geqq r$. Choose a path $\alpha$ joining $x$ and $y$ in $G$ such that $l(\alpha)<1+d_{1}(A)$. Since $f$ is locally $L$-bilipschitz, $l(f \alpha) \leqq L l(\alpha) \leqq L\left(1+d_{1}(A)\right)$. Hence

$$
|f(x)-f(y)| \leqq L\left(1+d_{1}(A)\right) \leqq L\left(1+d_{1}(A)\right)|x-y| / r .
$$

Consequently, $f$ is $L_{2}$-lipschitz with $L_{2}=\max \left(L, L\left(1+d_{1}(A)\right) / r\right)$.

\section{Deformation of embeddings near the identity}

3.1. In this section we present Sullivan's version of the Edwards-Kirby theory. Let $M$ be a manifold, possibly with boundary. If $X \subset M$, an embedding $f: X \rightarrow M$ is said to be clean if $f^{-1} \partial M=X \cap \partial M$. The family of all clean embeddings $f: X \rightarrow M$ is denoted by $E(X ; M)$. If $A \subset X$, we set

$$
E(X, A ; M)=\{f \in E(X ; M): f \mid A=\mathrm{id}\} .
$$

We first give a quantitative canonical Schoenflies theorem for embeddings near id.

3.2. Lemma. Let $A=\bar{B}^{n} \backslash B^{n}(1 / 2)$, and let

$$
\mathscr{P}=\left\{f \in E\left(A ; R^{n}\right): d(f, \mathrm{id})<1 / 30\right\} .
$$

Then there exist a continuous map $\varphi: \mathscr{P} \rightarrow E\left(\bar{B}^{n} ; R^{n}\right)$, a universal constant $a_{0}$, and a constant $b_{n}$ depending only on $n$ such that:

(1) $\varphi(f)\left|S^{n-1}=f\right| S^{n-1}$ for all $f \in \mathscr{P}$.

(2) $\varphi$ (id) $=\mathrm{id}$.

(3) If $f$ is $K$-QC, $\varphi(f)$ is $b_{n} K^{3}-\mathrm{QC}$.

(4) If $f$ is locally L-bilipschitz, $\varphi(f)$ is locally $a_{0} L^{3}$-bilipschitz.

(5) If $f$ is L-bilipschitz, $\varphi(f)$ is $a_{0} L^{3}$-bilipschitz. 
Proof. The map $\varphi(f)$ is constructed by the furling idea of M. Brown. We can follow the proof of $\left[\mathrm{GVm}_{1}\right.$, Lemma 9, p. 241]. However, since we do not assume that $S^{n-1} \subset \operatorname{im} e$, we choose $q=0.9$, and define the radial map $\lambda$ by $\lambda[0, b]=[0, a]$, $\lambda[b, q]=[a, q], \lambda[q, \infty]=[q, \infty]$. Moreover, we must have $2 c=b / a$ in order that the construction be valid in the LIP category. If we choose $a=0.6, b=0.8, c=2 / 3$ and $d=0.65$, the construction is possible for all $e \in \mathscr{P}$. Then $\varphi=(e \mapsto \hat{e})$ satisfies (1), (3), (4) and (5). Replacing $\varphi(e)$ by $\varphi(e) \varphi(\text { id })^{-1}$, we find $\varphi$ satisfying all conditions of the lemma.

3.3. Lemma. (Straightening of handles near id.) Let $m \geqq 0$ and $k \geqq 0$ be integers such that $m+k=n \geqq 2$. Then there exist a neighborhood $\mathscr{P}$ of id in $E\left(I^{n}, I^{m} \times \partial I^{k} ; R^{m} \times I^{k}\right)$ and a continuous map

$$
\psi: \mathscr{P} \times I \rightarrow E\left(I^{n}, I^{m} \times \partial I^{k} ; R^{m} \times I^{k}\right)
$$

with the following properties:

(1) $\psi(\mathrm{id}, t)=\mathrm{id}$ for all $t \in I$.

(2) $\psi(h, 0)=h$ for all $h \in \mathscr{P}$.

(3) $\psi(h, t)\left|\partial I^{n}=h\right| \partial I^{n}$ for all $h \in \mathscr{P}$ and $t \in I$.

(4) $\psi(h, 1) \mid\left(I^{m} \times \partial I^{k}\right) \cup\left(\bar{I}^{m}(1 / 2) \times I^{k}\right)=$ id for all $h \in \mathscr{P}$.

(5) If $h \in \mathscr{P}$ is $K-\mathrm{QC}$, then $\psi(h, t)$ is $K_{1}-\mathrm{QC}$ with $K_{1}$ depending only on $K$ and $n$.

(6) If $h \in \mathscr{P}$ is locally L-bilipschitz, then $\psi(h, t)$ is locally $L_{1}$-bilipschitz with $L_{1}$ depending only on $L$ and $n$.

(7) If $h \in \mathscr{P}$ is $L$-bilipschitz, then $\psi(h, t)$ is $L_{1}$-bilipschitz with $L_{1}$ depending only on $L$ and $n$.

Proof. Recall the notation $D^{m}=B_{\sigma}\left(0, r_{m}\right)=B^{m}\left(s_{m}\right)$ from 2.9. With the aid of an auxiliary bilipschitz homeomorphism of $R^{n}$, we may replace the cubes $I^{m}$ and $I^{k}$ by closed unit balls $\bar{B}^{m}$ and $\bar{B}^{k}$, and the cubes $\bar{I}^{m}(1 / 2)$ of (4) by the ball $\bar{B}_{\sigma}\left(0, r_{m} / 2\right)$. Performing a preliminary deformation given by [EK, Proposition 3.2], it suffices to consider embeddings $h \in E\left(\bar{B}^{m} \times \bar{B}^{k}, \bar{B}^{m} \times \partial B^{k} ; R^{m} \times \bar{B}^{k}\right)$ such that $h=$ id in $C=B^{m}(9 / 10) \times\left(\bar{B}^{k} \backslash B^{k}(1 / 2)\right)$. This deformation multiplies the dilatation and the bilipschitz constant of $h$ at most by a factor depending only on $n$. Let $u: A_{k}^{n} \rightarrow$ $B^{m} \times \bar{B}^{k}$ be the homeomorphism defined in 2.10. We first show that for every $h \in\left(\bar{B}^{m} \times \bar{B}^{k}, \bar{B}^{m} \times \partial B^{k} \cup C ; R^{m} \times \bar{B}^{k}\right)$ which is sufficiently close to the identity, there is a homeomorphism $g: R^{n} \rightarrow R^{n}$, continuously dependent on $h$, such that (i) $g=$ id if $h=\mathrm{id}$, (ii) $g=u^{-1} h u$ in $u^{-1}\left[\bar{D}^{m} \times \bar{B}^{k}\right]$, (iii) $g=$ id in $R^{n} \backslash B^{n}$, and (iv) $g$ respects the QC and bilipschitz properties of $h$.

If $m=0, h: \bar{B}^{n} \rightarrow \bar{B}^{n}$ is a homeomorphism with $h \mid S^{n-1}=\mathrm{id}$. Hence we can choose $g=h \cup\left(\mathrm{id} \mid R^{n} \backslash B^{n}\right)$. Suppose that $m \geqq 1$. Let us first recall the Sullivan covering map $\pi_{0}: B^{m} \rightarrow Q^{m}$ from 2.9. It is injective in a neighborhood of the ball $\bar{D}^{m}$. Choose a point $p \in Q^{m} \backslash \pi_{0} \bar{D}^{m}$ and a LIP immersion $\alpha_{0}: Q^{m} \backslash p \rightarrow B^{m}(9 / 10)$. By the LIP Schoenflies theorem [LuV, 7.7], we may assume that $\alpha_{0} \pi_{0}=\mathrm{id}$ in a neighborhood of $\bar{D}^{m}$. Set $\alpha=\alpha_{0} \times \mathrm{id}:\left(Q^{m} \backslash p\right) \times \bar{B}^{k} \rightarrow \bar{B}^{m} \times \bar{B}^{k}$. Then $\alpha e=u$ in a neigh- 
borhood of $u^{-1}\left[\bar{D}^{m} \times \bar{B}^{k}\right]$. Choose $t>0$ such that $4 t<\min \left(r_{m}, \sigma_{Q}\left(p, \pi_{0} \bar{D}^{m}\right)\right)$, and set $D_{j}=B_{\sigma_{Q}}(p, j t)$ for $j \leqq 4$. We can now construct the following variation of the main diagram of Edwards-Kirby.

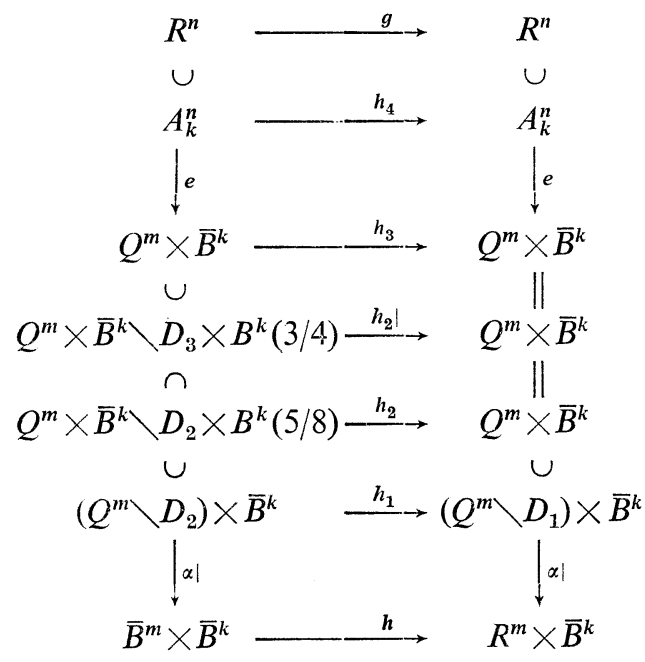

We start from the bottom with $h$ in a neighborhood $\mathscr{P}$ of id. On each step, we may replace $\mathscr{P}$ by a smaller neighborhood. We first find $h_{1}$ as in [EK, p. 68]. The embedding $h_{2}$ is the extension of $h_{1}$ via the identity, and the homeomorphism $h_{3}$ is found by the canonical Schoenflies theorem 3.2. The homeomorphism $h_{4}$ is the lifting $h_{3}^{*}$ given by 2.13 , and $g$ is the extension of $h_{4}$ via the identity. If $\mathscr{P}$ is a sufficiently small neighborhood of id, the properties (i), (ii) and (iii) are easily verified.

If $K \geqq 1$ or $L \geqq 1$, we let $K_{1}, K_{2}, \ldots$ or $L_{1}, L_{2}, \ldots$ denote constants depending only on $K$ and $n$ or $L$ and $n$, respectively. Suppose that $h$ is $K$-QC. We divide $Q^{m} \times \bar{B}^{k}$ into the following three sets:

$$
F_{1}=\left(Q_{m} \backslash \bar{D}_{3}\right) \times \bar{B}^{k}, \quad F_{2}=\bar{D}_{3} \times \bar{B}^{k}(3 / 4), \quad F_{3}=\bar{D}_{3} \times\left(\bar{B}^{k} \backslash B^{k}(3 / 4)\right) .
$$

Set $E_{j}=e^{-1} F_{j}, j=1,2,3$. Since $Q^{m}$ is compact, we can choose a set $U$, open in $A_{k}^{n}$, such that $\bar{U}$ is compact in $A_{k}^{n}$ and such that $e U=Q^{m} \times \bar{B}^{k}$. Then $\alpha e \mid U \backslash e^{-1}\left[\bar{D}_{1} \times \bar{B}^{k}\right]$ is locally $L_{1}$-bilipschitz in the euclidean metric. Since $\alpha e h_{4}=h \alpha e$ in $E_{1}, h_{4} \mid E_{1} \cap U$ is $K_{1}$-QC with $K_{1}=K L_{1}^{4 n-4}$. Since $h_{4}$ is compatible with $G^{m}$ by 2.14 and since $G^{m}$ acts conformally on $A_{k}^{n}, h_{4} \mid E_{1}$ is $K_{1}$-QC.

The set $E_{2}$ has a countable number of components $E_{2 j}, j \in N$, and $e$ maps each $E_{2 j}$ homeomorphically onto $F_{2}$. In each $E_{2 j}, h_{4}$ is constructed by Lemma 3.2 using an auxiliary bilipschitz embedding which is defined in a neightorhood of $E_{2 j}$ and which maps $E_{2 j}$ onto $\bar{B}^{n}$. Hence the dilatation of $h_{4} \mid E_{2 j}$ depends only on $K$, $n$ and $j$. However, if $i \neq j$, there is $g \in G^{m}$ such that $h_{4}\left|E_{2 i}=g h_{4} g^{-1}\right| E_{2 j}$. Hence $h_{4} \mid E_{2}$ is $K_{2}$-QC. 
Finally, $h_{4} \mid E_{3}=$ id, which implies that $h_{4}$ is $K_{3} \cdot \mathrm{QC}$ with $K_{3}=\max \left(K_{1}, K_{2}\right)$, cf. [V $\left.\ddot{a}_{1}, 35.1\right]$. Hence $g$ is $K_{3}$-QC.

Suppose that $h$ is locally $L$-bilipschitz. Arguing as in the QC case we conclude that $h_{4}$ is locally $L_{1}$-bilipschitz in the hyperbolic metric of $\bar{R}^{n} \backslash S^{m-1}$. On the other hand, $\sigma\left(h_{4}\right.$, id) $\leqq M_{n}$ by 2.14 . It follows from 2.12 that $h_{4}$ is (globally) $L_{2}$-bilipschitz in the euclidean metric. Hence $g$ is $L_{2}$-bilipschitz.

Define the Alexander isotopy $g_{t}: R^{n} \rightarrow R^{n}$ by $g_{t}(x)=\operatorname{tg}(x / t)$ for $0<t \leqq 1$, $g_{0}=$ id. Choose a bilipschitz embedding $b: \bar{B}^{n} \rightarrow \bar{B}^{m}(3 / 4) \times \bar{B}^{k}$ such that $v=u$ in $u^{-1}\left[\bar{B}^{m}(1 / 2) \times \bar{B}^{k}\right]$. Let $f_{t}: R^{n} \rightarrow R^{n}$ be the isotopy defined by $f_{t}=v g_{t}^{-1} v^{-1}$ in $v \bar{B}^{n}$ and by $f_{t}=$ id outside $v \bar{B}^{n}$. The desired deformation $\psi$ is obtained by setting

$$
\psi(h, t)=f_{t} h: \bar{B}^{m} \times \bar{B}^{k} \rightarrow R^{m} \times \bar{B}^{k} .
$$

If $h$ is in a sufficiently small neighborhood $\mathscr{P}$ of id, $\psi(h, t)$ is in

$$
E\left(\bar{B}^{n} \times \bar{B}^{k}, \bar{B}^{m} \times \partial B^{k} ; R^{m} \times \bar{B}^{k}\right),
$$

and the properties (1)-(7) of Lemma 3.3 are easily checked (remember that $\bar{D}^{m} \subset$ $\left.B^{m}(1 / 2)\right)$.

3.4. Theorem. Let $G$ be open in $\bar{R}^{n}$, and let $A, A^{\prime}, B, B^{\prime}, C, C^{\prime}$ be subsets of $G$ satisfying the following conditions: $A$ and $C$ are closed in $G, B$ is compact, and $A \subset \operatorname{int} A^{\prime}, B \subset$ int $B^{\prime}, C \subset \operatorname{int} C^{\prime}$. Then there exist a neighborhood $\mathscr{P}$ of id in $E\left(G, C^{\prime} ; \bar{R}^{n}\right)$, an open neighborhood $A^{\prime \prime} \subset A^{\prime}$ of $A$ in $G$ and a continuous map $\psi: \mathscr{P} \times$ $I \rightarrow E\left(G, C ; \bar{R}^{n}\right)$ such that the following conditions are satisfied for all $t \in I$ and $h \in \mathscr{P}$ :

(1) $\psi($ id, $t)=$ id.

(2) $\psi(h, 0)=h$.

(3) $\psi(h, t)=h$ in $G \backslash B^{\prime}$.

(4) $\psi(h, 1) \mid B \cup C=\mathrm{id}$.

(5) If $h$ is LQC in int $A^{\prime}, \psi(h, t)$ is LQC in $A^{\prime \prime}$. If $h \mid \operatorname{int} A^{\prime}$ is $K$-QC, $\psi(h, t) \mid A^{\prime \prime}$ is $K_{1}$-QC with $K_{1}$ depending only on $K$ and $n$.

(6) If $G \subset R^{n}$ and if $h \mid A^{\prime}$ is a LIP embedding, then $\psi(h, t) \mid A^{\prime \prime}$ is a LIP embedding. If $h \mid A^{\prime}$ is locally L-bilipschitz, $\psi(h, t) \mid A^{\prime \prime}$ is locally $L_{1}$-bilipschitz with $L_{1}$ depending only on $L$ and $n$.

(7) If $G \subset R^{n}$ and if $h \mid A^{\prime}$ is L-bilipschitz, $\psi(h, t) \mid A^{\prime \prime}$ is $L_{1}$-bilipschitz with $L_{\mathbf{1}}$ depending only on $L$ and $n$.

Proof. We may assume that $\infty \notin B$. Choose a compact PL manifold $P \subset$ int $B^{\prime}$ such that $B \subset$ int $P$ and a fine handle decomposition of $P$. As in [EK, pp. 71-73], we can find a neighborhood $\mathscr{P}$ and a map $\psi$ satisfying (1) - (4) by successive applications of Lemma 3.3, starting with zero handles. In order that (5) - (7) also be true, we must choose $P$ and the handle decomposition in a regular manner. For example, we can start with the cell decomposition $T_{0}$ of $R^{n}$ consisting of all cubes with vertices in $2^{k} Z^{n}$ and with side length $2^{k}$, where $k$ is a small negative integer. We let $P$ be the underlying polyhedron of a finite subcomplex $T_{1}$ of $T_{0}$ such that $B \subset$ int $P$ and such that $Q \subset B^{\prime}$ whenever $Q \in T_{0}$ and $Q$ meets $P$. Then 
$P \subset$ int $B^{\prime}$. Choose a simplicial subdivision $T_{2}$ of $T_{1}$ without new vertices [RS, 2.9]. Then the second barycentric subdivision $T_{2}^{\prime \prime}$ defines a handle decomposition $\mathscr{H}$ of $P$ as in [RS, p. 82]. Let $i(H)$ be the index of a handle $H \in \mathscr{H}$. Define $H \leqq H^{\prime}$ if $H \cap H^{\prime} \neq \emptyset$ and $i(H) \leqq i\left(H^{\prime}\right)$. Then $H \leqq H^{\prime} \leqq H^{\prime \prime}$ implies $H \leqq H^{\prime \prime}$. Let $\mathscr{H}_{0}$ be the set of the handles $H \in \mathscr{H}$ such that there is $H^{\prime} \in \mathscr{H}$ with $H \leqq H^{\prime}$ and $H^{\prime} \cap C \neq \emptyset$. We may assume that $P_{0}=\cup \mathscr{H}_{0} \subset C^{\prime}$. Let $H_{1}, \ldots, H_{s}$ be the handles of $\mathscr{H}_{\mathcal{C}} \backslash \mathscr{H}_{0}$ arranged in order of increasing index. For each $j$ we choose a PL embedding $\mu_{j}: R^{n} \rightarrow$ $B^{\prime} \backslash C$ such that im $\mu_{j}$ is a small neighborhood of $H_{j}$ and such that for $i\left(H_{j}\right)=k$, $m=n-k$ and $H_{j}^{*}=\mu_{j} I^{n}$ we have:

(i) $\mu_{j}\left[\bar{I}^{m}(1 / 2) \times I^{k}\right]=H_{j}$.

(ii) $\mu_{j}\left[R^{m} \times I^{k}\right] \cap\left(P_{0} \cup H_{1} \cup \ldots \cup H_{j-1}\right)=\mu_{j}\left[R^{m} \times \partial I^{k}\right]$.

(iii) There are similarity maps $\alpha_{j}: R^{n} \rightarrow R^{n}$ such that the embeddings $\alpha_{j} \mu_{j}$ belong to a finite set depending only on $n$.

The desired isotopy $\psi(h, t)$ is a composition $\varphi_{s} * \ldots * \varphi_{1}$, where

$$
\begin{aligned}
& \varphi_{j}(h, t)=\mu_{j} \psi_{k}\left(\mu_{j}^{-1} h \mu_{j} \mid I^{n}, t\right) \mu_{j}^{-1} \text { in } H_{j}^{*}, \\
& \varphi_{j}(h, t)=h \quad \text { outside } H_{j}^{*},
\end{aligned}
$$

and $\psi_{k}$ is given by Lemma 3.3 with $k=i\left(H_{j}\right)$.

The conditions (5) - (7) follow from the corresponding conditions of Lemma 3.3. In (5) we also need the fact that a set of finite $(n-1)$-dimensional measure is removable for $K$-QC homeomorphisms [V $\left.\ddot{a}_{1}, 35.1\right]$. In (7) we use the following elementary lemma: Let $G \subset R^{n}$ be open, let $A \subset G$ be compact, and let $f: G \rightarrow R^{n}$ be an embedding such that $f \mid A$ and $f \mid G \backslash A$ are $L$-bilipschitz. Then $f$ is $L$-bilipschitz.

3.5. Theorem. In Theorem 3.4, the conditions (2), (3), (4) can be replaced by the following ones:

(2') $\psi(h, 0)=$ id.

(3') $\psi(h, t) \mid G \backslash B^{\prime}=$ id.

(4') $\psi(h, 1)=h$ in $B \cup C$.

Proof. We may assume that $B^{\prime}$ is compact. Choose a closed neighborhood $A_{1}$ of $A$ in int $A^{\prime}$ and a compact neighborhood $B_{1}$ of $B$ in int $B^{\prime}$. Apply 3.4 with $A$ and $B$ replaced by $A_{1}$ and $B_{1}$. We obtain a map $\psi_{0}: \mathscr{P} \times I \rightarrow E\left(G, C ; \bar{R}^{n}\right)$ and an open neighborhood $A_{0}^{\prime \prime} \subset A^{\prime}$ of $A$. Choose an open neighborhood $A^{\prime \prime}$ of $A$ with $\mathrm{cl}_{G} A^{\prime \prime} \subset A_{0}^{\prime \prime}$. Replacing $\mathscr{P}$ by a smaller neighborhood we may assume that $h B \subset B_{1}$ and that $h\left[A^{\prime \prime} \cap B^{\prime}\right] \subset \psi_{0}(h, t) A_{0}^{\prime \prime}$ for all $h \in \mathscr{P}$ and $t \in I$. Then the map $\psi(h, t)=\psi_{0}(h, t)^{-1} h$ has the required properties.

3.6. Theorem. Let $G \subset R^{n}$ be open, let $B \subset G$ be compact, let $B^{\prime} \subset G$ be a neighborhood of $B$, and let $\varepsilon>0$. Then there is $\delta>0$ such that for every LIP embedding $h: G \rightarrow R^{n}$ with $d(h, \mathrm{id}) \leqq \delta$ there is a LIP homeomorphism $h^{\prime}: R^{n} \rightarrow R^{n}$ such that

(1) $d\left(h^{\prime}\right.$, id $) \leqq \varepsilon$,

(2) $h^{\prime}|B=h| B$,

(3) $h^{\prime} \mid R^{n} \backslash B^{\prime}=$ id. 
Furthermore, if $h$ is $K$-QC, $h^{\prime}$ is $K_{1}-\mathrm{QC}$ with $K_{1}$ depending only on $K$ and $n$. If $h$ is locally L-bilipschitz, $h^{\prime}$ is $L_{1}$-bilipschitz with $L_{1}$ depending only on $L$ and $n$.

Proof. We may assume that $B^{\prime}$ is compact. We apply Theorem 3.5 with $A=$ $A^{\prime}=G$ and $C=C^{\prime}=\emptyset$ and obtain a map $\psi: \mathscr{P} \times I \rightarrow E\left(G ; R^{n}\right)$. Since $\psi(\mathrm{id}, t)=\mathrm{id}$ for every $t \in I$, we may assume, replacing $\mathscr{P}$ by a smaller neighborhood, that $|\psi(h, t)(x)-x|<\varepsilon$ for all $h \in \mathscr{P}, t \in I$ and $x \in G$. There is $\delta>0$ such that $h \in \mathscr{P}$ whenever $h \in E\left(G ; R^{n}\right)$ and $d(h$, id $) \leqq \delta$. We can then define $h^{\prime}$ by $h^{\prime} \mid G=\psi(h, 1)$ and by $h^{\prime} \mid R^{n} \backslash G=$ id.

3.7. Remark. The qualitative part of Theorem 3.6 is the same as $\left[\mathrm{TV}_{2}, 2.19\right]$. This is all that was needed from Sullivan's theory in the proof of the QC extension theorem from dimension $n$ to $n+1$. However, it is possible to simplify this proof by making also use of the quantitative part of Theorem 3.6; see 7.1. We shall use this simplification in the proof of Theorem 7.4, which can be considered as a generalization of the result in $\left[\mathrm{TV}_{2}\right]$. For this purpose, we give a quantitative version of $\left[\mathrm{TV}_{2}, 2.20\right]$. For this and for later purposes, it is convenient to introduce the following notion:

3.8. Solid families. Let $X$ and $Y$ be metric spaces. A family $\mathscr{F}$ of embeddings $f: X \rightarrow Y$ is said to be solid if cl $\mathscr{F}$ is a compact family of embeddings. If $Y=R^{n}$ and if $X \subset R^{n}$ is either open or compact, $\mathscr{F}$ is solid if and only if it is pointwise bounded, equicontinuous and inversely equicontinuous. More precisely:

(1) For every $x_{0} \in X$, the set $\left\{f\left(x_{0}\right): f \in \mathscr{F}\right\}$ is bounded.

(2) For every $x_{0} \in X$ and $\varepsilon>0$, there is a neighborhood $U$ of $x_{0}$ such that $\left|f(x)-f\left(x_{0}\right)\right|<\varepsilon$ whenever $x \in U$ and $f \in \mathscr{F}$.

(3) For every $x_{0} \in X$ and for every neighborhood $U$ of $x_{0}$, there is $\varepsilon^{\prime}>0$ such that $\left|f(x)-f\left(x_{0}\right)\right| \geqq \varepsilon^{\prime}$ whenever $x \in X \backslash U$ and $f \in \mathscr{F}$.

Indeed, by Ascoli's theorem, (1) and (2) are true if and only if cl $\mathscr{F}$ is compact in $C\left(X, R^{n}\right)$, and (3) means that the members of $\mathrm{cl} \mathscr{F}$ are embeddings.

The corresponding statement is true if we replace $R^{n}$ by $\bar{R}^{n}$ and the euclidean metric by the spherical metric. However, in this case the condition (1) is superfluous.

Let $U \subset R^{n}$ be open and let $E_{\mathrm{LIP}}\left(U ; R^{n}\right)$ be the family of all LIP embeddings $f: U \rightarrow R^{n}$. By a result of Sullivan [Su], $E_{\mathrm{LIP}}\left(U ; R^{n}\right)$ is dense in $E\left(U ; R^{n}\right)$ if $n \neq 4$. We shall prove this in Section 4 (Corollary 4.5). We say that an embedding $f: U \rightarrow R^{n}$ is LIP approximable if $f \in \mathrm{cl} E_{\mathrm{Lip}}\left(U ; R^{n}\right)$, which is always true if $n \neq 4$.

We next give a quantitative version of $\left[\mathrm{TV}_{2}, 2.20\right]$. This will be needed in Section 7 .

3.9. Lemma. Let $U, U^{\prime}, V, W$ be open sets in $R^{n}$ such that

$$
W \subset V \subset U, \quad \bar{U}^{\prime} \subset U, \quad \bar{W} \cap U \subset V,
$$


and $\bar{U}^{\prime}$ is compact. Let $\mathscr{F}$ be a solid family of embeddings $g: U \rightarrow R^{n}$. For $n=4$ we also assume that the members of $\mathscr{F}$ are LIP approximable. Let $\varepsilon>0, K \geqq 1$, $L \geqq 1$. Then there are $\delta>0, K^{\prime} \geqq 1, L^{\prime} \geqq 1$ with the followin properties:

Let $h: V \rightarrow R^{n}$ be a LIP embedding, and let $g \in \mathscr{F}$ be such that $d(g, h ; V) \leqq \delta$. Then there is a LIP embedding $h^{\prime}: U \rightarrow R^{n}$ such that

(1) $d\left(h^{\prime}, g ; U^{\prime}\right) \leqq \varepsilon$,

(2) $h^{\prime}=h$ in $W \cap U^{\prime}$,

(3) $h^{\prime} \mid U^{\prime}$ is $K^{\prime}$-QC if $h$ is $K$-QC,

(4) $h^{\prime} \mid U^{\prime}$ is $L^{\prime}$-bilipschitz if $h$ is locally L-bilipschitz.

Here $\delta$ depends only on $\tau=\left(U, U^{\prime}, V, W, \mathscr{F}, \varepsilon\right), K^{\prime}$ depends on $\tau$ and $K$, and $L^{\prime}$ depends on $\tau$ and $L$.

Proof. We shall closely follow the proof of $\left[\mathrm{TV}_{2}, 2.20\right]$. Choose open sets $A$ and $A_{1}$ such that $\bar{U}^{\prime} \subset A, \bar{A} \subset A_{1}, \bar{A}_{1} \subset U$ and $\bar{A}_{1}$ is compact. Since $\mathscr{F}$ is solid, there is $\varepsilon_{1}>0$ such that $|g(x)-g(y)| \leqq \varepsilon / 2$ whenever $g \in \mathscr{F},|x-y| \leqq \varepsilon_{1}$ and $x, y \in A$. Next, by Theorem 3.6, there is $\delta_{1}>0$ such that if $f: A \cap V \rightarrow R^{n}$ is a LIP embedding with $d(f$, id; $A \cap V) \leqq \delta_{1}$, then there is a LIP homeomorphism $f^{\prime}: R^{n} \rightarrow R^{n}$ such that

$$
d\left(f^{\prime}, \mathrm{id}\right) \leqq \varepsilon_{1}, \quad f^{\prime}=f \text { in } W \cap U^{\prime}, \quad f^{\prime} \mid R^{n} \backslash(A \cap V)=\text { id. }
$$

Finally, we choose $\delta>0$ such that $y \in g A_{1}$ and $\left|g^{-1}(x)-g^{-1}(y)\right| \leqq \delta_{1}$ whenever $g \in \mathscr{F}, x \in g A, y \in R^{n}$ and $|x-y| \leqq 3 \delta$. We show that the lemma is true with this $\delta$.

Since cl $\mathscr{F}$ is compact and since its memters are LIP approximable, there is a finite family $\mathscr{F}^{\prime}$ of LIP embeddings $U \rightarrow R^{n}$ such that for every $g \in \mathrm{cl} \mathscr{F}$ we can choose $g^{\prime} \in \mathscr{F}^{\prime}$ with $d\left(g^{\prime}, g ; A_{1}\right) \leqq \min (\varepsilon / 2, \delta / 2)$. The family $\mathscr{F}^{\prime}$ depends only on $\tau$. Hence there are $K_{0}$ and $L_{0}$, depending only on $\tau$, such that for every $g^{\prime} \in \mathscr{F}^{\prime}$, $g^{\prime} \mid A_{1}$ is $K_{0}$-QC and $L_{0}$-bilipschitz.

Let $h: V \rightarrow R^{n}$ and $g \in \mathscr{F}$ be as in the lemma. Since $d\left(g^{\prime}, g ; A_{1}\right) \leqq \delta / 2$ and $B(g A, 3 \delta) \subset g A_{1}$, we have $B(g A, 5 \delta / 2) \subset g^{\prime} A_{1}$, which implies $B\left(g^{\prime} A, 2 \delta\right) \subset g^{\prime} A_{1}$. Since $d\left(g^{\prime}, h ; A \cap V\right)<2 \delta$, we can define an embedding $f: A \cap V \rightarrow R^{n}$ by $f=g^{\prime-1} h \mid A \cap V$. Then $f[A \cap V] \subset A_{1}$. Moreover, $d(f$, id; $A \cap V) \leqq \delta_{1}$. To show this, let $x \in A \cap V$. Then $|g(f(x))-g(x)| \leqq \delta+\left|g^{\prime}(f(x))-g^{\prime}(x)\right|=\delta+\left|h(x)-g^{\prime}(x)\right| \leqq$ $3 \delta$. By the choice of $\delta$, this yields $|f(x)-x| \leqq \delta_{1}$.

If (a) $h$ is $K$-QC, $f$ is $K K_{0}$-QC. If (b) $h$ is locally $L$-bilipschitz, $f$ is locally $L L_{0}$ bilipschitz. By the choice of $\delta_{1}$, there is a LIP homeomorphism $f^{\prime}: R^{n} \rightarrow R^{n}$ satisfying (3.10). Moreover, by Theorem 3.6, $f^{\prime}$ is $K_{1}$-QC in case (a) and $L_{1}$-bilipschitz in case (b), with $K_{1}$ depending on $\tau$ and $K, L_{1}$ on $\tau$ and $L$. Then $h^{\prime}=g^{\prime}\left(f^{\prime} \mid U\right): U \rightarrow R^{n}$ is the desired embedding. In case (a) it is $K_{0} K_{1}-\mathrm{QC}$ and in case (b) $L_{0} L_{1}$-bilipschitz in $U^{\prime}$. In $W \cap U^{\prime}$ we have $h^{\prime}=g^{\prime} f^{\prime}=g^{\prime} f=h$, and thus (2) is true. Furthermore, $d\left(h^{\prime}, g ; U^{\prime}\right) \leqq d\left(g^{\prime} f^{\prime}, g f^{\prime} ; A\right)+d\left(g f^{\prime}, g ; A\right)$.

Since $f^{\prime} A=A, d\left(g^{\prime} f^{\prime}, g f^{\prime} ; A\right)=d\left(g, g^{\prime} ; A\right) \leqq \varepsilon / 2$. Since $d\left(f^{\prime}\right.$, id; $\left.A\right) \leqq \varepsilon_{1}$, the choice of $\varepsilon_{1}$ implies that $d\left(g f^{\prime}, g ; A\right) \leqq \varepsilon / 2$, and we obtain (1).

3.11. Stable homeomorphisms and the annulus conjecture. Let $C$ be one of the categories TOP, LQC, LIP. A $C$-homeomorphism $f: R^{n} \rightarrow R^{n}$ is said to be $C$-stable 
if $f$ can be written as a composite map $f_{1} \ldots f_{k}$ where each $f_{j}: R^{n} \rightarrow R^{n}$ is a $C$-homeomorphism such that $f_{j} \mid U_{j}=\mathrm{id}$ for some open $U_{j} \neq \emptyset \quad[\mathrm{BG}]$. The stable homeomorphism conjecture for $C$ states that every sense-preserving $C$-homeomorphism $f: R^{n} \rightarrow R^{n}$ is $C$-stable. Sullivan [Su] proved this for $C=\mathrm{LQC}$ and LIP in all dimensions; we give this in 3.12 below. For the case $n \leqq 3$, see also [Vä ${ }_{3}$ ] and [Kk, 4.5 and 4.6]. For $C=$ TOP, the conjecture is known to be true for $n \neq 4$ by Kirby and Siebenmann; the case $n=4$ is unsolved.

To formulate the annulus conjecture we need some terminology. A set $S \subset R^{n}$ is an $(n-1)$-dimensional $C$-sphere if there is a $C$-homeomorphism $f: S \rightarrow S^{n-1}$. For $C=\mathrm{LQC}$ this means that $f$ is quasisymmetric in the sense of $\left[\mathrm{TV}_{1}\right]$. We say that $S$ is locally $C$-flat if every point $x \in S$ has a neighborhood $U \subset R^{n}$ such that there is a $C$-homeomorphism $h:(U, U \cap S) \rightarrow\left(B^{n}, B^{n-1}\right)$. By a basic flattening theorem, every locally $C$-flat $(n-1)$-dimensional $C$-sphere $S \subset R^{n}$ is $C$-flat, that is, there is a $C$-homeomorphism $f:\left(R^{n}, S\right) \rightarrow\left(R^{n}, S^{n-1}\right)$. For $C=$ TOP this is a well-known result of M. Brown. For $C=\mathrm{LIP}$ it is given in [LuV, 7.8]. The case $C=\mathrm{LQC}$ follows from Theorem 4.10 and from the Schoenflies theorem.

The annulus conjecture for $C$ states that if $S_{1}$ and $S_{2}$ are locally $C$-flat $(n-1)$ dimensional $C$-spheres in $R^{n}$ and if $S_{1}$ lies in the bounded component of $R^{n} \backslash S_{2}$, then the closure of the domain bounded by $S_{1} \cup S_{2}$ is $C$-homeomorphic to the standard annulus $\bar{B}^{n} \backslash B^{n}(1 / 2)$. The stable homeomorphism conjecture implies the annulus conjecture; see [EG] or $[\mathrm{Kr}]$.

\subsection{Theorem. The stable homeomorphism conjecture and the annulus conjecture} are true in the categories $\mathrm{LQC}$ and LIP in all dimensions.

Proof. Let $C$ be either LQC or LIP, and let $\mathscr{F}$ be the set of all $C$-stable $C$-homeomorphisms $f: R^{n} \rightarrow R^{n}$. It suffices to show that $\mathscr{F}$ is the group $H_{C}^{+}\left(R^{n}\right)$ of all sensepreserving $C$-homeomorphisms $f: R^{n} \rightarrow R^{n}$. The case $k=0$ of Lemma 3.3 implies that id is an interior point of $\mathscr{F}$ in $H_{C}^{+}\left(R^{n}\right)$. Since $\mathscr{F}$ is a subgroup, it is both open and closed in $H_{C}^{+}\left(R^{n}\right)$. Hence it suffices to show that $H_{C}^{+}\left(R^{n}\right)$ is connected. We show that every $f \in H_{C}^{+}\left(R^{n}\right)$ can be joined by a path to id. There is $x_{0} \in R^{n}$ at which $f$ is differentiable with a positive jacobian. We may assume that $x_{0}=0=f\left(x_{0}\right)$. Set $g_{t}(x)=f(t x) / t$ for $t>0$ and $g_{0}=f^{\prime}(0)$. Then the path $t \mapsto g_{t}$ joins the linear map $f^{\prime}(0)$ to $f$. Since $\operatorname{det} f^{\prime}(0)>0$, it follows from elementary linear algebra that $f^{\prime}(0)$ and id can be joined by a path.

3.13. Conventions. In the rest of this section, $C$ will denote either LQC or LIP. We say that a homeomorphism $f: S^{n-1} \rightarrow S^{n-1}$ is LQC if it is quasisymmetric in the sense of $\left[\mathrm{TV}_{1}\right]$ and $\left[\mathrm{V}_{4}\right]$.

3.14. Theorem. Let $f: S^{n-1} \rightarrow S^{n-1}$ be a sense-presering C-homeomorphism and let $0<a<1$. Then $f$ can be extended to a $C$-homeomorphism $g: \bar{B}^{n} \rightarrow \bar{B}^{n}$ such that $g \mid B^{n}(a)=\mathrm{id}$. 
Proof. It follows easily from 3.12 that $f$ can be written as the composition $f_{1} \ldots f_{k}$ of $C$-homeomorphisms $f_{j}: S^{n-1} \rightarrow S^{n-1}$ such that each $f_{j}$ is the identity in some nonempty open subset of $S^{n-1}$. Hence we may assume that $f \mid V=$ id for some open nonempty $V \subset S^{n-1}$. It suffices to find an extension $g$ such that $g \mid U=\mathrm{id}$ for some open $U \subset B^{n}, U \neq \emptyset$. Indeed, we can then choose a $C$-homeomorphism $h: \bar{B}^{n} \rightarrow \bar{B}^{n}$ such that $B^{n}(a) \subset h U$ and $h \mid S^{n-1}=\mathrm{id}$, and the desired map is $h g h^{-1}$.

If $C=\mathrm{LIP}$, we let $g$ be the cone of $f$. Suppose that $C=\mathrm{LQC}$. By $\left[\mathrm{TV}_{2}, 3.15 .4\right]$, we can choose a QC extension $g_{1}: \bar{B}^{n} \rightarrow \bar{B}^{n}$ of $f$. Choose a QC homeomorphism $\varphi: R^{n} \rightarrow R^{n}$ such that $\varphi \mid S^{n-1} \backslash V=\mathrm{id}, B^{n} \subset \varphi B^{n}$ and $|\varphi(0)|>1$. Setting

$$
\begin{array}{lll}
g(x)=\varphi^{-1} g_{1} \varphi(x) & \text { if } & |\varphi(x)| \leqq 1, \\
g(x)=x & \text { if } & |\varphi(x)|>1,
\end{array}
$$

we obtain the required map $g: \bar{B}^{n} \rightarrow \bar{B}^{n}$.

3.15. Notation. For $0<a<b$ we let $A(a, b)$ denote the closed annulus $\bar{B}^{n}(b) \backslash B^{n}(a)$.

3.16. Theorem. Let $0<a<b$, let $0<r<b$, and let $f: A(a, b) \rightarrow R^{n}$ be a sensepreserving $C$-embedding such that the bounded component of $R^{n} \backslash f S^{n-1}(b)$ contains $\bar{B}^{n}(r) \cup f S^{n-1}(a)$. Then there is a $C$-embedding $g: \bar{B}^{n}(b) \rightarrow R^{n}$ such that $g=f$ near $\partial B^{n}(b)$ and $g \mid \bar{B}^{n}(r)=\mathrm{id}$.

Proof. Choose $r_{1}$ and $a_{1}$ such that $r<r_{1}<a_{1}<b, a<a_{1}$ and $\bar{B}^{n}\left(r_{1}\right) \cap$ $f A\left(a_{1}, b\right)=\emptyset$. Let $R$ be the domain bounded by $S^{n-1}\left(r_{1}\right) \cup f S^{n-1}\left(a_{1}\right)$. By the annulus theorem 3.12, there is a sense-preserving $C$-homeomorphism $h: A\left(r_{1}, a_{1}\right) \rightarrow \bar{R}$ with $h S^{n-1}\left(r_{1}\right)=S^{n-1}\left(r_{1}\right)$. By Theorem 3.14, $h^{-1} f \mid S^{n-1}\left(a_{1}\right)$ can be extended to a $C$-homeomorphism $\varphi: A\left(r_{1}, a_{1}\right) \rightarrow A\left(r_{1}, a_{1}\right)$ such that $\varphi \mid S^{n-1}\left(r_{1}\right)=$ id. Similarly, $h \mid S^{n-1}\left(r_{1}\right)$ can be extended to a $C$-homeomorphism $h_{1}: \bar{B}^{n}\left(r_{1}\right) \rightarrow \bar{B}^{n}\left(r_{1}\right)$ with $h_{1} \mid \bar{B}^{n}(r)=\mathrm{id}$. The desired $g: \bar{B}^{n}(b) \rightarrow R^{n}$ is obtained by setting

$$
\begin{array}{ll}
g(x)=f(x) & \text { if } \quad a_{1} \leqq|x| \leqq b, \\
g(x)=h(\varphi(x)) & \text { if } \quad r_{1} \leqq|x| \leqq a_{1}, \\
g(x)=h_{1}(x) & \text { if } \quad|x| \leqq r_{1} .
\end{array}
$$

3.17. Theorem. Let $0<a<b<c<d$ and let $f: A(a, b) \cup A(c, d) \rightarrow R^{n}$ be a sensepreserving $C$-embedding. Suppose also that $f A(a, b) \cup f S^{n-1}(c)$ lies in the bounded component of $R^{n} \backslash f S^{n-1}(d)$ and that $f S^{n-1}(a)$ lies in the bounded component of $R^{n} \backslash f S^{n-1}(b)$. Then there is a C-embedding $g: A(a, d) \rightarrow R^{n}$ such that $g=f$ near $\partial A(a, d)$.

Proof. Replacing $a$ by a slightly larger number and $d$ by a slightly smaller number, we may assume that $f S^{n-1}(a)$ and $f S^{n-1}(d)$ are locally $C$-flat. By the annulus theorem 3.12, we may assume that $|f(x)|=|x|$ for all $x \in \partial A(a, d)$. 
By Theorem 3.16, there is a $C$-homeomorphism $g_{1}: \bar{B}^{n}(d) \rightarrow \bar{B}^{n}(d)$ such that $g_{1}=f$ near $\partial B^{n}(d)$ and $g_{1}=$ id near $\partial B^{n}(a)$. Together with an auxiliary inversion, Theorem 3.16 also implies that there is a $C$-homeomorphism $g_{2}: R^{n} \backslash B^{n}(a) \rightarrow$ $R^{n} \backslash B^{n}(a)$ such that $g_{2}=f$ near $\partial B^{n}(a)$ and $g_{2}=$ id near $R^{n} \backslash B^{n}(d)$. Then $g=$ $g_{2} g_{1} \mid A(a, d)$ is the desired embedding.

\section{LQC and LIP approximation of homeomorphisms}

4.1. In this section we show that if $C=\mathrm{LIP}$ or LQC, and if $M$ and $N$ are $C$-manifolds without boundary of dimension $n \neq 4$, then every homeomorphism $f: M \rightarrow N$ can be approximated by $C$-homeomorphisms. We next prove the same result for manifolds with boundary provided that $n \neq 4,5$. In particular, we obtain the LIP and LQC hauptvermutung for these manifolds. For manifolds without boundary, these results are due to Sullivan [Su]; the LIP case for manifolds with boundary has been proved by J. Luukkainen (unpublished). The key of the proof is the following result whose PL analogue is true only for $k \neq 3$ (by Kirby and Siebenmann).

4.2. Lemma. (C-straightening of TOP handles.) Let $m \geqq 0$ and $k \geqq 0$ be integers with $m+k=n \geqq 2, n \neq 4$, let $C$ be either LQC or LIP, let $U$ be an open neighborhood of $I^{n}$, and let $f \in E\left(U ; R^{n}\right)$ be a $C$-embedding near $I^{m} \times \partial I^{k}$. Let $\varepsilon>0$. Then there is an isotopy $\varphi: I \rightarrow E\left(U ; R^{n}\right)$ such that:

(1) $\varphi_{0}=f$.

(2) $\varphi_{t}=f$ near $U \backslash$ int $I^{n}$ for all $t \in I$.

(3) $\left|\varphi_{t}(x)-f(x)\right| \leqq \varepsilon$ for all $t \in I$ and $x \in U$.

(4) $\varphi_{1}$ is a $C$-embedding near $I^{m} \times \partial I^{k} \cup \bar{I}^{m}(1 / 2) \times I^{k}$.

Proof. By the Schoenflies theorem, we may assume that $U=R^{n}$ and that $f$ is a homeomorphism onto $R^{n}$.

Choose $\delta \in(0,1)$ such that $|f(x)-f(y)| \leqq \varepsilon / 2$ whenever $x, y \in I^{n}(2)$ and $|x-y| \leqq \delta$. Then $\mathscr{P}_{1}=\left\{g \in H\left(R^{n}\right): d\left(g, \mathrm{id} ; I^{n}\right)<\delta\right\}$ is a neighborhood of id in $H\left(R^{n}\right)$. Choose $r>0$ such that $f$ is a $C$-embedding in $A^{\prime}=B^{n}\left(I^{m} \times \partial I^{k}, 2 r\right)$. Apply Theorem 3.4 with $G=R^{n}, A=\bar{B}^{n}\left(I^{m} \times \partial I^{k}, r\right), \quad B$ a compact neighborhood of $\bar{I}^{m}(1 / 2) \times \bar{I}^{k}(1-r)$ in int $I^{n}$, and $B^{\prime}$ a compact neighborhood of $B$ in int $I^{n}$. We obtain a neighborhood $\mathscr{P}$ of id in $H\left(R^{n}\right)$ and a map $\psi: \mathscr{P} \times I \rightarrow H\left(R^{n}\right)$. Since $\psi($ id, $t)=$ id, we may assume that $\operatorname{im} \psi \subset \mathscr{P}_{1}$, replacing $\mathscr{P}$ by a smaller neighborhood.

Since $n \neq 4$, there is a PL homeomorphism $g \in H\left(R^{n}\right)$ such that $d\left(g, f ; R^{n}\right)<$ $\varepsilon / 2$ and $g^{-1} f=h \in \mathscr{P}$. If $k=0$, we need here the deep stable homeomorphism theorem of Kirby-Siebenmann, unless $f$ is known to be stable. Set $h_{t}=\psi(h, t)$. We show that $\varphi_{t}=g h_{t}$ is the desired isotopy. First $\varphi_{0}=g h_{0}=g h=f$. Outside $B^{\prime}$ we have $\varphi_{t}=g h=f$. If $x \in B^{\prime}$, then $\left|h_{t}(x)-x\right|<\delta$, and we obtain

$$
\left|\varphi_{t}(x)-f(x)\right| \leqq\left|g\left(h_{t}(x)\right)-f\left(h_{t}(x)\right)\right|+\left|f\left(h_{t}(x)\right)-f(x)\right| \leqq \varepsilon / 2+\varepsilon / 2=\varepsilon .
$$


In $B, \varphi_{1}=g h_{1}=g$ is PL, hence a $C$-embedding. In $A, h_{t}$ is a $C$-embedding for every t. In particular, $\varphi_{1}\left|A=g h_{1}\right| A$ is a $C$-embedding. Consequently, $\varphi_{1}$ is a $C$-embedding in int $A \cup$ int $B$, which is a neighborhood of $I^{m} \times \partial I^{k} \cup \bar{I}^{m}(1 / 2) \times I^{k}$.

4.3. Notation. If $M$ and $N$ are $n$-manifolds without boundary, we let $E(M ; N)$ denote the set of all embeddings $f: M \rightarrow N$.

4.4. Theorem. Let $C$ be either LQC or LIP, let $M$ and $N$ be $n$-dimensional $C$-manifolds without boundary, $n \neq 4$, let $A, B \subset M$ be closed, and let $B^{\prime} \subset M$ be a neighborhood of $B$. Let $f: M \rightarrow N$ be an embedding such that $f$ is a $C$-embedding near $A$, and let $\varepsilon: M \rightarrow(0, \infty)$ be continuous. Then there is a continuous map $\varphi: I \rightarrow$ $E(M ; N)$ such that:

(1) $\varphi_{0}=f$.

(2) $\varphi_{t}=f$ in $M \backslash B^{\prime}$ and near $A$.

(3) $\varphi_{1}$ is a $C$-embedding near $A \cup B$.

(4) $d\left(\varphi_{t}(x), f(x)\right)<\varepsilon(x)$ for all $t \in I$ and $x \in M$, where $d$ is a given metric of $N$.

Proof. 1. Special case. $M$ is open in $R^{n}, N=R^{n}$, and $B$ is compact. Choose an open neighborhood $A^{\prime}$ of $A$ such that $f \mid A^{\prime}$ is a $C$-embedding. Choose a compact PL manifold $P \subset$ int $B^{\prime}$ such that $B \subset$ int $P$. Choose a fine handle decomposition $\mathscr{H}$ of $P$ as in [RS, p. 82]. Let $i(H)$ denote the index of a handle $H \in \mathscr{H}$. Define $H \leqq H^{\prime}$ if $H \cap H^{\prime} \neq \emptyset$ and $i(H) \leqq i\left(H^{\prime}\right)$. Then $H \leqq H^{\prime} \leqq H^{\prime \prime}$ implies $H \leqq H^{\prime \prime}$. Let $\mathscr{H}_{0}$ be the set of the handles $H \in \mathscr{H}$ such that there is $H^{\prime} \in \mathscr{H}$ with $H \leqq H^{\prime}$ and $H^{\prime} \cap A \neq \emptyset$. We may assume that $P_{0}=\cup \mathscr{H}_{0} \subset A^{\prime}$. Let $H_{1}, \ldots, H_{s}$ be the handles of $\mathscr{H} \backslash \mathscr{H}_{0}$ arranged in order of increasing index. For each $j$ we choose a PL embedding $\mu_{j}: R^{n} \rightarrow B^{\prime} \backslash A$ such that for $i\left(H_{j}\right)=k, m=n-k$ and $H_{j}^{*}=\mu_{j} I^{n}$ we have

$$
\begin{gathered}
\mu_{j}\left[\bar{I}^{m}(1 / 2) \times I^{k}\right]=H_{j}, \\
H_{j}^{*} \cap\left(P_{0} \cup H_{1} \cup \ldots \cup H_{j-1}\right)=\mu_{j}\left[I^{m} \times \partial I^{k}\right] .
\end{gathered}
$$

We may assume that $H_{j}^{*} \cap H_{k}^{*}=\emptyset$ whenever $i\left(H_{j}\right)=i\left(H_{k}\right)$ and $j \neq k$.

The isotopy $\varphi$ is defined in $s$ steps as follows: Set $f_{0}=f$, and suppose that the $(j-1)^{\text {th }}$ step has produced an embedding $f_{j-1}: M \rightarrow N$. We apply Lemma 4.2 with the substitution $f \mapsto f_{j-1} \mu_{j}$ and with a small $\varepsilon$. This yields an isotopy $\varphi^{\prime}: I \rightarrow E\left(R^{n} ; R^{n}\right)$. Define an isotopy $\varphi^{j}: I \rightarrow E(M ; N)$ by $\varphi_{t}^{j}=\varphi_{t}^{\prime} \mu_{j}^{-1}$ in $H_{j}^{*}$ and by $\varphi_{t}^{j}$ elsewhere. Combining the isotopies $\varphi^{1}, \ldots, \varphi^{s}$ gives the desired $\varphi$.

2. General case. Let $A^{\prime}$ be as in the special case. Choose a closed $B_{0} \subset B \backslash A$ such that $\mathrm{cl}\left(B \backslash B_{0}\right) \subset A^{\prime}$. Choose a locally finite covering $\mathscr{B}$ of $B_{0}$ by open subsets of $M$ such that for every $W \in \mathscr{B}, W$ and $f W$ can be $C$-embedded in $R^{n}$ and $\bar{W}$ is compact in $B^{\prime} \backslash A$. By [Mu, 2.7] we may assume that $\mathscr{B}$ can be expressed as $\mathscr{B}_{0} \cup$ $\ldots \cup \mathscr{B}_{n}$, where each collection $\mathscr{B}_{j}$ is disjoint. For every $W \in \mathscr{B}$ we choose a smaller open set $W^{\prime}$ such that $\bar{W}^{\prime} \subset W$ and such that the sets $W^{\prime}$ cover $B_{0}$. The isotopy 
$\varphi$ is constructed in $n+1$ steps so that the $j^{\text {th }}$ step is active only in $\cup \mathscr{B}_{j}$. We give the first two steps. Set $E=\operatorname{cl}\left(B \backslash B_{0}\right)$.

Let $W \in \mathscr{B}_{0}$. We apply the special case with the substitution $f \mapsto f \mid W, A \mapsto E \cap W$, $B \mapsto \bar{W}^{\prime}, B^{\prime} \mapsto$ a compact neighborhood of $\bar{W}^{\prime}$ in $W$, and with a small $\varepsilon$. This yields an isotopy of $f \mid W$ for every $W \in \mathscr{B}_{0}$. These isotopies can be extended by $f \mid M \backslash\left(\cup \mathscr{B}_{0}\right)$ to an isotopy of $f$, which produces an embedding $f_{1}: M \rightarrow N$.

In the second step, we take $W \in \mathscr{B}_{1}$ and apply the special case with $f_{\mapsto} \mapsto f_{1} \mid W$, $A \mapsto\left(E \cup\left(\cup\left\{\bar{W}^{\prime}: W \in \mathscr{B}_{0}\right\}\right)\right) \cap W, B \mapsto \bar{W}^{\prime}$, and $B^{\prime}$ and $\varepsilon$ as above.

4.5. Corollary. Let $M$ and $N$ be n-dimensional C-manifolds without boundary, $n \neq 4$, and let $\varepsilon: M \rightarrow(0, \infty)$ be continuous. Then for every homeomorphism $f: M \rightarrow N$ there is a C-homeomorphism $g: M \rightarrow N$ such that $d(f(x), g(x))<\varepsilon(x)$ for all $x \in M$. In particular, the hauptvermutung is true for these manifolds in the categories LQC and LIP.

4.6. Theorem. Every topological $n$-manifold without boundary, $n \neq 4$, has a LIP manifold structure (and hence an LQC manifold structure).

Proof. The theorem follows in a well-known manner from Theorem 4.4. See, for example, [Kr, Theorem 10].

4.7. Lemma. Let $C$ be either LQC or LIP. Let $A$ be closed in $B^{n-1}$, let $B \subset B^{n-1}$ be compact, and let $f: B_{+}^{n} \rightarrow R_{+}^{n}$ be a clean embedding such that $f \mid B^{n-1}$ is a $C$ embedding and such that $f$ is a $C$-embedding near $A$ in $B_{+}^{n}$. Then, for every $\varepsilon>0$ there is an isotopy $\varphi: I \rightarrow E\left(B_{+}^{n} ; R_{+}^{n}\right)$ such that:

(1) $\varphi_{0}=f$.

(2) $\varphi_{t}\left|B^{n-1}=f\right| B^{n-1}$ for every $t \in I$.

(3) For some $b \in(0,1), \varphi_{t}=f$ in $B_{+}^{n} \backslash B_{+}^{n}(b)$ for every $t \in I$.

(4) $\varphi_{1}$ is a $C$-embedding near $A \cup B$.

(5) $\left|\varphi_{t}(x)-f(x)\right|<\varepsilon$ for all $x \in B_{+}^{n}$ and $t \in I$.

Proof. Choose numbers $0<b_{1}<b_{2}<b<1$ such that $B \subset B^{n-1}\left(b_{1}\right)$. By the Schoenflies theorem, we can extend $f \mid B^{n-1}(b)$ to a $C$-homeomorphism $g: R^{n-1} \rightarrow$ $R^{n-1}$ such that $g$ is QC if $C=\mathrm{LQC}$. For $n=2$ this follows from [ $\mathrm{LeV}_{2}$, II, Lemma 7.2], Let $F: R_{+}^{n} \rightarrow R_{+}^{n}$ be a $C$-homeomorphism with $F \mid R^{n-1}=g$. For $C=L I P$, we may choose $F=g \times$ id; for $C=\mathrm{LQC}$ we apply $\left[\mathrm{TV}_{2}, 3.12\right]$. Then $W=F^{-1} f B_{+}^{n}(b)$ is a neighborhood of $\bar{B}^{n-1}\left(b_{2}\right)$ in $R_{+}^{n}$. Choose $r>0$ and set $a_{j}=\left(r^{2}+b_{j}^{2}\right)^{1 / 2}$ and $V_{j}=B^{n}\left(-r e_{n}, a_{j}\right) \cap R_{+}^{n}$ for $j=1,2$. Then $V_{2} \subset W$ if $r$ is large enough. Let $\alpha: I \rightarrow$ $E\left(R_{+}^{n} \backslash \bar{B}^{n-1}\left(b_{1}\right) ; R_{+}^{n} \backslash \bar{B}^{n-1}\left(b_{1}\right)\right)$ be the obvious vertical isotopy through LIP embeddings such that $\alpha_{0}=\mathrm{id}, \alpha_{t} \mid R_{+}^{n} \backslash V_{2}=\mathrm{id}$, and im $\alpha_{1}=R_{+}^{n} \backslash \bar{V}_{1}$. Then it is easy to verify that the lemma is true for a sufficiently large $r$ and with $\varphi$ as follows:

$$
\begin{aligned}
& \varphi_{t}=F \alpha_{t} F^{-1} f \alpha_{t}^{-1} \text { in } B_{+}^{n} \cap\left(\operatorname{im} \alpha_{t}\right) \\
& \varphi_{t}=F \quad \text { in } \quad B_{+}^{n} \backslash \operatorname{im} \alpha_{t} . \quad \square
\end{aligned}
$$


4.8. Theorem. Theorem 4.4, Corollary 4.5 and Theorem 4.6 are true for $n$-manifolds with boundary, $n \neq 4,5$. In Theorem $4.4, E(M ; N)$ denotes the set of all embeddings $g: M \rightarrow N$ such that $g^{-1} \partial N=\partial M$, and we assume that $f \in E(M ; N)$. If $f \mid \partial M$ is a C-embedding, Theorem 4.4 is also true for $n=5$, and we can choose $\varphi$ so that $\varphi_{t}|\partial M=f| \partial M$ for all $t \in I$.

Proof. We prove the special case of 4.4; the rest of the proof is essentially unchanged. We assume that in $4.4 M$ is open in $R_{+}^{n}$, that $N=R_{+}^{n}$ and that $B$ is compact. We may assume that $B^{\prime}$ is compact. Choose an open neighborhood $A^{\prime}$ of $A$ such that $f \mid A^{\prime}$ is a $C$-embedding. Applying 4.4 to $f_{0}=f \mid M \cap R^{n-1}$ (for $n=2$ see Remark 4.9) we find a small isotopy $\varphi^{\prime}: I \rightarrow E\left(M \cap R^{n-1} ; R^{n-1}\right)$ of $f_{0}$ which is active only in a compact neighborhood $D$ of $\left(B \cap R^{n-1}\right) \backslash A^{\prime}$ in (int $\left.B^{\prime} \cap R^{n-1}\right) \backslash A$ and which produces an embedding $\varphi_{1}^{\prime}: M \cap R^{n-1} \rightarrow R^{n-1}$ which is a $C$-embedding near $(A \cup B) \cap R^{n-1}$. Using a spindle neighborhood of $D$ in $M, \varphi^{\prime}$ can be extended in a well-known manner to a small isotopy $\varphi: I \rightarrow E(M ; N)$ of $f$ such that $\varphi_{t}=f$ in $M \backslash B^{\prime}$ and near $A$ for all $t \in I$. If $f \mid \partial M$ is a $C$-embedding, we let $\varphi$ be the constant isotopy.

Choose a covering of $\left(B \cap R^{n-1}\right) \backslash A^{\prime}$ by half balls $U_{1}, \ldots, U_{s}$ such that $\bar{U}_{j} \subset$ $B^{\prime} \backslash A$ and such that $\varphi_{1} \mid U_{j} \cap R^{n-1}$ is a $C$-embedding. Àpplying Lemma $4.7 \mathrm{~s}$ times gives a small isotopy $\psi: I \rightarrow E(M ; N)$ of $\varphi_{1}$ which is active only in $U_{1} \cup \ldots \cup U_{s}$, satisfies $\psi_{t}\left|\partial M=\varphi_{1}\right| \partial M$ for all $t \in I$, and produces a map $\psi_{1}$ which is a $C$-embedding near $A \cup\left(B \cap R^{n-1}\right)$ in $M$.

Choose compact $B_{1}, B_{2}$ such that $B=B_{1} \cup B_{2}, B_{1} \subset$ int $R_{+}^{n}$, and $\psi_{1}$ is a $C$ embedding near $B_{2}$. Apply 4.4 with the substitution $M \mapsto \operatorname{int} M, N \mapsto$ int $R_{+}^{n}$, $A \mapsto\left(A \cup B_{2}\right) \cap$ int $M, B \mapsto B_{1}, B^{\prime} \mapsto$ a compact neighborhood of $B_{1}$ in $B^{\prime} \cap$ int $M$, $f \mapsto \psi_{1} \mid$ int $M$. We obtain an isotopy $\chi$ of $\psi_{1}$, and the desired isotopy is the combination $\chi * \psi * \varphi$.

4.9. Remark. Suppose that $n=2$. Then in the proof of 4.8 we need the special case of Theorem 4.4 for $n=1$. For $C=\mathrm{LIP}$ this is very easy. We prove the case $C=\mathrm{LQC}$. We may assume that $M \subset R^{1}$ is an open interval, that $B$ is a compact subinterval, that $N=R^{1}$ and that $f$ is increasing. Replacing $A$ by a closed neighborhood, we may assume that $A$ is the union of a locally finite family of closed intervals. Replacing $B$ by a finite family of disjoint subintervals and treating each of these separately, we may assume that $B=[0,1], B^{\prime}=[-1,2]$, and that $B^{\prime} \cap A$ is either empty or $[-1, r]$ or $[-1, r] \cup[1-r, 2]$ for some $r \in(0,1 / 2)$. It suffices to find an embedding $f_{1}: M \rightarrow R^{1}$ such that (1) $f_{1}=f$ in $M \backslash B^{\prime}$ and near $A$, (2) $f_{1}$ is LQC near $A \cup B$ and (3) $d\left(f_{1}, f ; B^{\prime}\right)<\varepsilon$. Indeed, we can then define $\varphi_{t}=$ $(1-t) f+t f_{1}$.

Suppose, for example, that $B^{\prime} \cap A=[-1, r]$. Choose $s \in(r, 1 / 2)$ such that $f$ is QC near [0,s]. There is a QC extension $g: R^{1} \rightarrow R^{1}$ of $f[[0, s]$ such that $g$ is $C^{1}$ outside [0,s]; see [LeV 1, p. 12]. Choose $s_{1} \in(s, 1 / 2)$ such that $g\left(s_{1}\right)<f(3 / 2)$. Define $f_{1}: M \rightarrow R^{1}$ as follows: $f_{1}(x)=f(x)$ if $x \leqq s$ or $x \geqq 3 / 2, f_{1}(x)=g(x)$ if 
$s \leqq x \leqq s_{1}$, and $f_{1}$ is a suitable PL function on $\left[s_{1}, 3 / 2\right]$. Then $f_{1}$ satisfies (1) and (2), and (3) can easily be arranged.

4.10. Theorem. Let $M$ and $N$ be LQC $n$-manifolds and let $f: \partial M \rightarrow \partial N$ be an LQC embedding. Then $f$ can be extended to an LQC embedding of a neighborhood of $\partial M$.

Proof. Since $\partial M$ and $\partial N$ have TOP collars, $f$ can be extended to an embedding of a neighborhood of $\partial M$. If $n \neq 4$, the theorem follows directly from the last statement of Theorem 4.8. However, since the last isotopy $\chi$ of the proof of Theorem 4.8 is not needed in the present case, the theorem is also true for $n=4$.

4.11. Remark. The LIP version of 4.10 is clear, since the boundary of a LIP manifolds has a LIP collar [LuV, 7.5]. The concept "LQC collar" seems to be meaningless, since there is no natural way to form products in the category LQC, cf. [ $\mathrm{Tu}_{2}$, Example 4]. Theorem 4.10 acts as a substitute for collaring in the LQC manifold theory.

\section{Extension}

5.1. In this section we consider the following problem: Let $G$ be open in $\bar{R}^{n}$, and let $U \subset G$ be an open set such that $U \cup \partial G$ is a neighborhood of $\partial G$ in $\bar{G}$. Suppose that $f: G \rightarrow \bar{R}^{n}$ is an embedding such that $f \mid U$ is $K$-QC. Does there exist an embedding $g: G \rightarrow \bar{R}^{n}$ such that $g=f$ near $\partial G$ and such that $g$ is $K^{\prime}$-QC with some $K^{\prime}$ depending only on $G, U$ and $K$ ? The following example shows that without any restrictions the answer is negative: Let $G=B^{n}(4) \backslash \bar{B}^{n}, U=\left(B^{n}(2) \backslash \bar{B}^{n}\right) \cup$ $\left(B^{n}(4) \backslash \bar{B}^{n}(3)\right)$. For $0<a<1$ we let $f: G \rightarrow R^{n}$ be a radial embedding such that $f(x)=a x$ for $1<|x|<2$ and $f(x)=x$ for $3<|x|<4$. Then $f \mid U$ is $1-\mathrm{QC}$, but the dilatation of $g$ must converge to $\infty$ as $a \rightarrow 0$.

We shall show that the answer is positive if $U$ is connected. Moreover, for $n=4$ we must also assume that $f$ is QC. In Theorem 5.7 we shall show that the connectedness condition can be replaced by a condition concerning the size of the boundary components of $f G$. As special cases, we obtain quantitative versions of the Schoenflies theorem and the annulus theorem.

Corresponding results are also true in the category LIP.

5.2. Lemma. Let $G \subset \bar{R}^{n}$ be open, let $f: G \rightarrow \bar{R}^{n}$ be an embedding and let $g: G \rightarrow \bar{R}^{n}$ be an immersion such that $f=g$ near $\partial G$. Then $g G=f G$, and $g$ is an embedding.

Proof. We may assume that $G$ is connected and that $f$ and $g$ are defined on $\bar{G}$. Then the topological degree $\mu(y, f, G)$ is \pm 1 for $y \in f G$ and $\mu(y, f, G)=0$ for $y \in \bar{R}^{n} \backslash f \bar{G}$. Moreover, $\mu(y, f, G)=\mu(y, g, G)$ for all $y \in \bar{R}^{n} \backslash f \partial G$ and $\mu(y, g, G)=$ \pm card $g^{-1}(y)$. See, for example, [RR, § II.2]. The lemma follows. 
In the following theorem, all closures and boundaries are taken in $\bar{R}^{n}$ :

5.3. Theorem. Let $G$ be a domain in $\bar{R}^{n}$, let $U$ and $V$ be open in $G$ such that $U \cup \partial G$ and $V \cup \partial G$ are neighborhoods of $\partial G$ in $\bar{G}, \bar{V} \cap G \subset U$, and $U$ is connected. Let $f: G \rightarrow \bar{R}^{n}$ be an embedding such that $f \mid U$ is $K$-QC and such that either $n \neq 4$ or $f$ is QC. Then there is a $K^{\prime} \cdot \mathrm{QC}$ embedding $g: G \rightarrow \bar{R}^{n}$ such that $g|V=f| V$ and $K^{\prime}$ depends only on $G, U, V$ and $K$.

Proof. Choose a connected open set $U_{1} \subset G$ such that $\bar{V} \cap G \subset U_{1}$ and $\bar{U}_{1} \cap$ $G \subset U$. Then $C=\partial U_{1} \cap G$ is a compact subset of $U \backslash \bar{V}$. Choose open sets $G_{1}$, $G_{2}, G_{3}, G_{4}$ such that $\bar{G}_{i+1} \subset G_{i}, C \subset G_{4}$, and $\bar{G}_{1} \subset U \backslash \bar{V}$. We apply Theorem 3.4 with the substitution $G \mapsto G_{2}, A \mapsto G_{2}, A^{\prime} \mapsto G_{2}, B \mapsto \bar{G}_{4}, B^{\prime} \mapsto G_{3}, K \mapsto K^{2}$. We obtain a neightorhood $\mathscr{P}$ of id in $E\left(G_{2} ; \bar{R}^{n}\right)$, a continuous map $\psi: \mathscr{P} \times I \rightarrow E\left(G_{2} ; \bar{R}^{n}\right)$ and a number $K_{1} \geqq K$, depending only on $K$ and $n$. Letting $q$ denote the spherical metric of $\bar{R}^{n}$ we choose $\varepsilon_{1}>0$ such that the set

$$
N_{q}\left(\mathrm{id}, G_{2}, \varepsilon_{1}\right)=\left\{f \in E\left(G_{2} ; \bar{R}^{n}\right): q(f(x), x)<\varepsilon_{1} \text { for all } x \in G_{2}\right\}
$$

is contained in $\mathscr{P}$. Set $\varepsilon=\min \left(\varepsilon_{1}, q\left(G_{2}, \partial G_{1}\right)\right)$, and fix three distinct points $a_{1}$, $a_{2}, a_{3}$ in $U$. Let $\mathscr{F}$ be the family of all $K$-QC embeddings $u: U \rightarrow \bar{R}^{n}$ such that (1) $u\left(a_{j}\right)=a_{j}$ for $j=1,2,3$, and (2) $u$ has an extersion to a QC embedding $v: G \rightarrow \bar{R}^{n}$. Then $\mathscr{F}$ depends only on $G, U$ and $K$. Since $U$ is connected, $\mathscr{F}$ is solid (3.8 and [V $\ddot{a}_{1}, 19.5$ and 21.1]). Hence there is $\varepsilon^{\prime}>0$ such that $q(u(x), u(y)) \geqq \varepsilon^{\prime}$ whenever $x, y \in \bar{G}_{1}, q(x, y) \geqq \varepsilon$ and $u \in \mathscr{F}$. Furthermore, there is a finite family $\left\{u_{1}, \ldots, u_{s}\right\} \subset \mathscr{F}$ such that $\mathscr{F}$ is covered by the sets $N_{q}\left(u_{j}, \bar{G}_{1}, \varepsilon^{\prime}\right), 1 \leqq j \leqq s$. For each $j \in[1, s]$ we choose a QC extension $v_{j}: G \rightarrow \bar{R}^{n}$ of $u_{j}$. Choose $K_{0}=K_{0}(G, U, K)$ such that each $v_{j}$ is $K_{0}$-QC.

Let $f: G \rightarrow \bar{R}^{n}$ be an embedding such that $f \mid U$ is $K$-QC.

Case 1. $f$ is QC. We show that $g$ can be chosen to be $K_{0} K_{1}$-QC. By an auxiliary Möbius transformation we may assume that $f\left(a_{j}\right)=a_{j}$ for $j=1,2,3$. Then $f \mid U \in \mathscr{F}$. Hence $f \in N_{q}\left(u_{j}, \bar{G}_{1}, \varepsilon^{\prime}\right)$ for some $j \in[1, s]$. Since $q\left(u_{j} \bar{G}_{2}, u_{j} \partial G_{1}\right) \geqq \varepsilon^{\prime}, f G_{2} \subset u_{j} G_{1}$. Setting $h=u_{j}^{-1} f \mid G_{2}$ we thus obtain a $K^{2}$-QC emtedding $h: G_{2} \rightarrow G_{1}$, which belongs to $N_{q}\left(\mathrm{id}, G_{2}, \varepsilon\right)$ and hence to $\mathscr{P}$. Then the $K_{\mathrm{I}}$-QC embedding $h_{1}=\psi(h, 1): G_{2} \rightarrow \bar{R}^{n}$ satisfies the conditions $h_{1} \mid \bar{G}_{4}=\mathrm{id}$ and $h_{1}\left|G_{2} \backslash G_{3}=h\right| G_{2} \backslash G_{3}$. Define $g: G \rightarrow \bar{R}^{n}$ as follows:

$$
\begin{array}{lll}
g(x)=f(x) & \text { for } & x \in U_{1} \backslash \bar{G}_{3}, \\
g(x)=u_{j}\left(h_{1}(x)\right) & \text { for } & x \in\left(G_{2} \cap U_{1}\right) \cup G_{4}, \\
g(x)=v_{j}(x) & \text { for } & x \in G \backslash \bar{U}_{1} .
\end{array}
$$

Then $g$ is a well-defined immersion. Since $g=f$ in $V, g$ is an embedding by Lemma 5.2. Since $g$ is $K_{0} K_{1}-\mathrm{QC}$, Case 1 is proved.

Case 2. $n \neq 4$. Applying Theorem 4.4 with the substitution $M \mapsto G, A \mapsto \widetilde{U}_{1} \cap G$, $B \mapsto \mathrm{cl}\left(G \backslash U_{1}\right), B^{\prime} \mapsto G \backslash V, f \mapsto f$, we obtain a QC embedding $\varphi_{1}: G \rightarrow \bar{R}^{n}$ such 
that $\varphi_{1}\left|U_{1}=f\right| U_{1}$ is $K$-QC. Hence this case reduces to Case 1 with $U$ replaced by $U_{1}$.

5.4. Quantitative QC Schoenflies theorem. Let $0<r<1$, and let $f: \bar{B}^{n} \backslash B^{n}(r) \rightarrow \bar{R}^{n}$ be a K-QC embedding. Then there is a $K^{\prime}$-QC embedding $g: \bar{B}^{n} \rightarrow \bar{R}^{n}$ such that $g\left|\partial B^{n}=f\right| \partial B^{n}$ and $K^{\prime}$ depends only on $K, n$ and $r$.

Proof. Choose $s \in(r, 1)$. By the QC Schoenflies theorem, there is a QC embedding $f_{1}: \bar{B}^{n} \rightarrow \bar{R}^{n}$ such that $f_{1}(x)=f(x)$ for $s \leqq|x| \leqq 1$. The theorem follows from 5.3.

5.5. Remark. Theorem 5.4 was proved by M. Näätänen [Nä] in the special case where $f \partial B^{n}$ is a sphere. A shorter proof for this case was given by D. B. Gauld and M. K. Vamanamurthy $\left[\mathrm{GVm}_{2}\right]$. If $n \geqq 3$, it follows from the extension theorem of $\left[\mathrm{TV}_{2}\right]$ that $K^{\prime}$ can in this case be chosen to be independent of $r$ [TV $\left.2,3.14\right]$.

5.6. Nonconnected $U$. Suppose that $U$ in Theorem 5.3 is not connected. Let $\mathscr{F}_{0}$ be the family of all $K \cdot \mathrm{QC}$ embeddings $u: U \rightarrow \bar{R}^{n}$ which have a QC extension $v: G \rightarrow \bar{R}^{n}$. In the proof of 5.3 we used the normalization $u\left(a_{j}\right)=a_{j}$, which gave a solid subfamily $\mathscr{P} \subset \mathscr{F}_{0}$. This is not possible if $U$ is not connected. However, there are other restrictions which give rise to solid families. As an example we prove the following result:

5.7. Theorem. Let $G, U, V$ be as in Theorem 5.3 except that $U$ is not necessarily connected. Let $\alpha>0$ and let $f: G \rightarrow \bar{R}^{n}$ be an embedding such that $f \mid U$ is $K$-QC and such that either $n \neq 4$ or $f$ is QC. Suppose that for each component $U_{1}$ of $U$, the spherical diameter of $\left(\mathrm{cl} f U_{1}\right) \backslash f G$ is at least $\alpha$. Then there is a $K^{\prime}$-QC embedding $g: G \rightarrow \bar{R}^{n}$ such that $g|V=f| V$ and $K^{\prime}$ depends only on $G, U, V, K$ and $\alpha$.

Proof. By Theorem 5.3 we may assume that $U$ is not connected. Let $\mathscr{F}$ be the family of all $K$-QC embeddings $u: U \rightarrow \bar{R}^{n}$ such that $u$ has a QC extension $v: G \rightarrow \bar{R}^{n}$ and such that for each component $U_{1}$ of $U, q\left(\left(\operatorname{cl} u U_{1}\right) \backslash u G\right) \geqq \alpha$ where $q$ is the spherical metric of $\bar{R}^{n}$. It suffices to show that $\mathscr{F}$ is solid, since the proof of 5.3 will then be valid also in the present case.

From $\left[V \ddot{a}_{1}, 19.2\right]$ it follows that $\mathscr{F}$ is equicontinuous, and hence cl $\mathscr{F}$ is compact in $C\left(U, \bar{R}^{n}\right)$. Suppose that $\left(f_{j}\right)$ is a sequence in $\mathscr{F}$ converging to a map $f: U \rightarrow \bar{R}^{n}$ uniformly on compact sets. Let $U_{1}$ be a component of $U$. Then $f \mid U_{1}$ is either constant or an embedding $\left[V \ddot{a}_{1}, 21.3\right]$. Assume that $f \mid U_{1}$ is constant. Choose a compact set $A \subset U_{1}$ such that no component of $\bar{R}^{n} \backslash A$ meets both $\partial U_{1} \cap G$ and $\partial U_{1} \backslash G$. Then for every $j$, no component of $\bar{R}^{n} \backslash f_{j} A$ meets both $\partial f_{j} U_{1} \cap f_{j} G$ and $\partial f_{j} U_{1} \backslash f_{j} G$. Hence there are disjoint sets $W_{1}$ and $W_{2}$ which are unions of components of $\bar{R}^{n} \backslash f_{j} A$ such that $q\left(W_{1}\right) \geqq \alpha$ and $q\left(W_{2}\right) \geqq \alpha$. This contradicts the fact that the maps $f_{j} \mid A$ converge uniformly to a constant. Hence $f \mid U_{1}$ is an embedding for every com. ponent $U_{1}$ of $U$. From this it easily follows that $f$ is an embedding. 
5.8. Quantitative QC annulus theorem. Suppose that in Theorem 3.17, $f: A(a, b) \cup A(c, d) \rightarrow R^{n}$ is $K$-QC and that $d\left(f S^{n-1}(d)\right) / d\left(f S^{n-1}(a)\right) \leqq M$. Then $g: A(a, d) \rightarrow R^{n}$ can be chosen to be $K^{\prime}$-QC with $K^{\prime}$ depending only on $a, b, c, d$, $M, K$ and $n$.

Proof. Using an auxiliary similarity map of $R^{n}$ we may assume that $0 \in \operatorname{im} f$ and that $d\left(f S^{n-1}(d)\right)=1$. Thus $d\left(f S^{n-1}(a)\right) \geqq 1 / M$. Choose $a_{1} \in(a, b)$ and $d_{1} \in(c, d)$. By Theorem 3.17, there is a QC embedding $f_{1}: A(a, d) \rightarrow R^{n}$ such that $f_{1}=f$ in $A\left(a, a_{1}\right) \cup A\left(d_{1}, d\right)$. The theorem now follows directly from Theorem 5.7 .

5.9. Lipschitz versions. There are several ways to formulate the results of this section in the category LIP. First, one can consider either $L$-bilipschitz or locally $L$-bilipschitz embeddings. Second, one can use either the euclidean or the spherical metric. The proofs are fairly easy modifications of the proofs given in the QC case. As an example we give the bilipschitz version of Theorem 5.4:

5.10. Quantitative bilipschitz Schoenflies theorem. Let $0<r<1$ and let $f: \bar{B}^{n} \backslash B^{n}(r) \rightarrow R^{n}$ be an L-bilipschitz embedding such that $f S^{n-1}(r)$ is contained in the bounded component of $R^{n} \backslash f S^{n-1}$. Then there is an $L^{\prime}$-bilipschitz embedding $g: \bar{B}^{n} \rightarrow R^{n}$ such that $g\left|\partial B^{n}=f\right| \partial B^{n}$ and $L^{\prime}$ depends only on $L, n$ and $r$.

\section{Quasihyperbolic metric and solid homeomorphisms}

6.1. This section is a preparation for Section 7. We give some properties of the quasihyperbolic metric of a domain in $R^{n}$. This metric has been considered in [GP], [GO] and [Vu]. Our work partially overlaps with [Vu]. We also introduce the solid homeomorphisms which are more general than QC homeomorphisms but which share certain common properties with the latter class of maps.

6.2. Quasihyperbolic metric. In the rest of Section $6, D$ and $D^{\prime}$ will always denote proper subdomains of $R^{n}$, where $n \geqq 2$ is a fixed integer. For $x \in D$ we set

$$
\varrho_{D}(x)=d(x, \partial D)^{-1}
$$

The quasihyperbolic metric $k_{D}$ of $D$ is defined by the element of length $\varrho_{D}(x)|d x|$. This means that for $a, b \in D$ we have

$$
k_{D}(a, b)=\inf \int_{\alpha} \varrho_{D} d s
$$

where the infimum is taken over all rectifiable paths $\alpha$ joining $a$ and $b$ in $D$. If $\partial D$ is an affine subspace $T$ of $R^{n}, k_{D}$ is equal to the hyperbolic metric $\sigma_{T}$ of $D$, defined in 2.4 . 
Gehring and Osgood [GO, Lemma 1] proved that each pair of points $a, b$ in $D$ can be joined by a quasihyperbolic geodesic $\alpha$, that is,

$$
k_{D}(a, b)=\int_{\alpha} \varrho_{D} d s
$$

If $\emptyset \neq A \subset D$ with $\bar{A}$ compact in $D$, we define the relative size of $A$ in $D$ as the number

$$
r_{D}(A)=\frac{d(A)}{d(A, \partial D)}
$$

Observe that if $r_{D}(A)<2$ and if $a, b \in A$, the segment $a b$ lies in $D$. Moreover, if $r_{D}(A)<1$, then $A$ is contained in a ball in $D$.

We start with the following elementary and well-known property of $R^{n}$ :

6.3. Lemma. Let $E \subset R^{n}$ be a bounded set, and let $t>0$ be such that $|a-b| \geqq t$ whenever $a, b \in E$ and $a \neq b$. Then card $E \leqq \varkappa_{n}(d(E) / t)$ where $\varkappa_{n}(\alpha)=(1+\alpha \sqrt{n})^{n}$.

Proof. Choose a closed cube $Q$ of side length $d(E)$ such that $E \subset Q$. Let $k$ be the integer for which $k-1 \leqq d(E) \sqrt{n} / t<k$. Divide $Q$ into $k^{n}$ closed cubes $Q_{i}$ of side length $d(E) / k$. Then $d\left(Q_{i}\right)<t$, and thus each $Q_{i}$ contains at most one point of $E$. Hence card $E \leqq k^{n}$.

6.4. Lemma. Let $\mu>0$ and let $A \subset D$ be a compact connected set with $r_{D}(A) \leqq \mu$. Then there is a constant $c=c(\mu, n) \geqq 1$ such that each pair of points $a, b \in A$ can be joined by a path $\alpha$ in $D$ such that $l(\alpha) \leqq c|a-b|$. In fact, one can choose $c=\chi_{n}(2+2 \mu) / 4$ for all $\mu$ and $c=1$ for $\mu<2$.

Proof. Set $t=d(A, \partial D)$, and let $a, b \in A$. If $|a-b|<2 t$, we can join $a$ and $b$ by a line segment in $D$. Suppose that $|a-b| \geqq 2 t$. Since $A$ is connected, the neighborhood $B(A, t / 2)$ is arcwise connected, Choose an arc $C$ joining $a$ and $b$ in $B(A, t / 2)$. Let $\leqq$ be the natural ordering of $C$ with $a<b$. Define points $a=a_{0}<$ $a_{1}<\ldots<a_{s}=b$ of $C$ by $a_{j+1}=\max \left(C \cap \bar{B}^{n}\left(a_{j}, t / 2\right)\right)$. Then $\left|a_{i}-a_{j}\right| \geqq t / 2$ whenever $0 \leqq i<j \leqq s-1$. Furthermore, $d(C) \leqq d(A)+t \leqq(1+\mu) t$. Hence Lemma 6.3 implies $s \leqq \varkappa_{n}(2+2 \mu)$. Let $\alpha$ be the broken line with vertices $a_{0}, a_{1}, \ldots, a_{s}$. Then $\alpha$ is in $D$, and

$$
l(\alpha) \leqq s t / 2 \leqq \chi_{n}(2+2 \mu)|a-b| / 4 .
$$

6.5. Lemma. Let $\mu>0$ and let $A \subset D$ be compact and connected with $r_{D}(A) \leqq \mu$. Then there is $c_{1}=c_{1}(\mu, n) \geqq 1$ such that

$$
\frac{1}{c_{1}} \leqq \frac{k_{D}(a, b) d(A, \partial D)}{|a-b|} \leqq c_{1}
$$

whenever $a, b \in A$ and $a \neq b$. Moreover, $\lim _{\mu \rightarrow 0} c_{1}(\mu, n)=1$, and $c_{1}(\mu, n)$ is increasing in $\mu$. 
Proof. Set $M=d(A)$ and $t=d(A, \partial D)$. Define $r=r(\mu) \in(0,1)$ by

$$
r=\min (\mu, 1 / 2) \text {. }
$$

Let $D^{\prime}$ be the neighborhood $B(A, r t)$. Then $M \leqq \mu t$ and $r_{D^{\prime}}(A) \leqq \mu / r$. By Lemma 6.4, there is a path $\alpha$ joining $a$ and $b$ in $D^{\prime}$ such that $l(\alpha) \leqq c|a-b|$ with $c=c(\mu / r, n)$. Then

$$
k_{D}(a, b) \leqq \int_{\alpha} \varrho_{D} d s \leqq \int_{\alpha} \frac{d s}{(1-r) t} \leqq \frac{c|a-b|}{(1-r) t} .
$$

This proves the second inequality of 6.5 with $c_{1}=c /(1-r)$. For $\mu \leqq 1 / 2$ we have $c_{1}=1 /(1-\mu)$, and hence $c_{1} \rightarrow 1$ as $\mu \rightarrow 0$.

To prove the first inequality, let $\alpha$ be a rectifiable path joining $a$ and $b$ in $D$. If $\operatorname{im} \alpha \subset D^{\prime}$, then

$$
\int_{\alpha} \varrho_{D} d s \geqq \int_{\alpha} \frac{d s}{t+M+r t} \geqq \frac{|a-b|}{(1+\mu+r) t} .
$$

If $\operatorname{im} \alpha \neq D^{\prime}, \alpha$ has two subpaths $\alpha_{1}, \alpha_{2}$ joining $A$ and $D \backslash D^{\prime}$ in $D^{\prime}$. Since $|a-b| \leqq$ $M \leqq \mu t$, we obtain

$$
\int_{\alpha} \varrho_{D} d s \geqq \int_{\alpha_{1} \cup \alpha_{2}} \frac{d s}{t+M+r t} \geqq \frac{2 r}{1+\mu+r} \geqq \frac{2 r|a-b|}{(1+\mu+r) \mu t} .
$$

Together with (6.6) this gives the first inequality of 6.5 with

$$
c_{1}=(1+\mu+r) \max (1, \mu / 2 r) \text {. }
$$

For $\mu \leqq 1 / 2$ we have $c_{1}=1+2 \mu$, and hence $c_{1} \rightarrow 1$ as $\mu \rightarrow 0$.

6.7. Corollary. The quasihyperbolic metric of $D$ is LIP equivalent to the euclidean metric.

6.8. Remark. Since $c_{1}(\mu, n) \rightarrow 1$ as $\mu \rightarrow 0$, the identity map $i:\left(D, k_{D}\right) \rightarrow(D, d)$ is locally almost 1 -quasisymmetric in the following sense: For every $x \in D$ and $\varepsilon>0$ there is a neighborhood $U$ of $x$ such that $i \mid U$ is $\eta$-quasisymmetric in the sense of $\left[\mathrm{TV}_{1}\right]$ with $\eta(t)=(1+\varepsilon) t$.

Lemma 6.5 also shows that $r_{D}(A)$ cannot be much smaller than the quasihyperbolic diameter $k_{D}(A)$ of $A$. More precisely, we have the following result:

6.9. Lemma. Let $A \subset D$ be compact. Then

If, in addition, $A$ is connected,

$$
r_{D}(A) \leqq 2\left(e^{k_{D}(A)}-1\right) .
$$

$$
k_{D}(A) \leqq c_{1}\left(r_{D}(A), n\right) r_{D}(A) .
$$

Proof. The first inequality follows from the inequality

$$
k_{D}(a, b) \geqq \log \left(1+\varrho_{D}(a)|a-b|\right),
$$


proved in [GP, (2.2)]. The second inequality follows directly from 6.5. See also [Vu, 2.18 and 2.19. (4)].

6.10. Solid homeomorphisms. A homeomorphism $f: D \rightarrow D^{\prime}$ is said to be solid, if $f$ and $f^{-1}$ are uniformly continuous in the quasihyperbolic metrics of $D$ and $D^{\prime}$. We say that a homeomorphism $f: D \rightarrow D^{\prime}$ is $\varphi$-solid if $\varphi:[0, \infty) \rightarrow[0, \infty)$ is a homeomorphism and if

$$
\varphi^{-1}\left(k_{D}(a, b)\right) \leqq k_{D^{\prime}}(f(a), f(b)) \leqq \varphi\left(k_{D}(a, b)\right)
$$

for all $a, b \in D$. From the convexity of the quasihyperbolic metric it easily follows that $f$ is solid if and only if $f$ is $\varphi$-solid for some $\varphi$. Moreover for every $r>0$ one can choose $\varphi$ so that $\varphi \mid[r, \infty$ ) is linear (see the proof of Lemma 6.21).

F. W. Gehring and B. G. Osgood [GO, Theorem 3] proved that every $K$-QC homeomorphism $f: D \rightarrow D^{\prime}$ is $\varphi_{K}$-solid with

$$
\varphi_{K}(t)=c \max \left(t, t^{\alpha}\right),
$$

where $\alpha=K^{1 /(1-n)}$ and $c=c(K, n)$. On the other hand, a solid homeomorphism need not be QC. For example, if $f, g: D \rightarrow D^{\prime}$ are homeomorphisms such that $g$ is solid and $f=g$ outside a compact set, then $f$ is solid. The simple extension $F_{f}: R_{+}^{n+1} \rightarrow R_{+}^{n+1}$ of a QC map $f: R^{n} \rightarrow R^{n}$ considered in $\left[\mathrm{TV}_{2}\right]$ is solid in $H^{n+1}=$ int $R_{+}^{n+1}$ but not usually QC (see 7.1). The following theorem, which is closely related to a result of Gehring [Ge, Theorem 4], shows, however, that quasiconformality can be characterized in terms of solidity:

6.12. Theorem. A homeomorphism $f: D \rightarrow D^{\prime}$ is quasiconformal if and only if there is a homeomorphism $\varphi:[0, \infty) \rightarrow[0, \infty)$ such that the restriction of $f$ to every subdomain of $D$ is $\varphi$-solid.

Proof. If $f$ is $K$-QC and if $G \subset D$ is a subdomain, then $f \mid G$ is $K$-QC and hence $\varphi_{K}$-solid with $\varphi_{K}$ as in (6.11). Conversely, suppose that $f \mid G$ is $\varphi$-solid for every domain $G \subset D$. Let $x \in D$. For $r>0$ we let $L(r)$ and $l(r)$ denote the maximum and minimum of $|f(y)-f(x)|$ over $y \in S^{n-1}(x, r)=S_{r}$. Set $G=D \backslash x$ and $G^{\prime}=$ $D^{\prime} \backslash f(x)=f G$. Then for sufficiently small $r$ we have $r_{G}\left(S_{r}\right)=2$ and $L(r) / l(r) \leqq$ $r_{G^{\prime}}\left(f S_{r}\right)$. Since $k_{G^{\prime}}\left(f S_{r}\right) \leqq \varphi\left(k_{G}\left(S_{r}\right)\right)$, Lemma 6.9 implies that $L(r) / l(r)$ is bounded by a constant depending only on $\varphi$ and $n$. Hence $f$ is $K$-QC for some $K=K(\varphi, n)$ [V $\left.\ddot{a}_{1}, 34.2\right]$.

6.13. In the rest of this section we give alternative characterizations to the solidity of a homeomorphism in terms of solid families of embeddings (see 3.8). This explains the term. In Section 7 we show that for $n \neq 4$ solid homeomorphisms can be approximated by QC mappings which are bilipschitz in the quasihyperbolic metric. 
By a cube in $R^{n}$ we always mean a closed $n$-cube with sides parallel to the coordinate axes. For every cube $Q$, we let $z_{Q}$ be its center and $2 \lambda_{Q}$ its side length. Thus $Q=z_{Q}+\lambda_{Q} I^{n}$. Let $\alpha_{Q}: R^{n} \rightarrow R^{n}$ be the affine map

$$
\alpha_{Q}(x)=z_{Q}+\lambda_{Q} x \text {. }
$$

Thus $\alpha_{Q} I^{n}=Q$. For $t>0$ we let $Q(t)$ be the open cube $\alpha_{Q} I^{n}(t)=z_{Q}+\lambda_{Q} I^{n}(t)$; the corresponding closed cube is then denoted by $\bar{Q}(t)$.

If $D \subset R^{n}$ is a proper subdomain and if $1<r \leqq s$, we let $\mathscr{K}_{D}(r, s)$ denote the family of all cubes $Q \subset D$ such that $Q(r) \subset D$ and $\bar{Q}(s) \notin D$. Furthermore, we set $\mathscr{K}_{D}(r)=\mathscr{K}_{D}(r, r)$. If $Q \in \mathscr{K}_{D}(r, s)$ and if $1 \leqq t<r$, then obviously

$$
\frac{2 t}{s-t} \leqq r_{D}(\bar{Q}(t)) \leqq \frac{2 t \sqrt{n}}{r-t} .
$$

6.15. Lemma. Suppose that $1 \leqq t<r \leqq s$ and that $Q \in \mathscr{K}_{D}(r, s)$. Then $\alpha_{Q} \mid \bar{I}^{n}(t):\left(\bar{I}^{n}(t), d\right) \rightarrow\left(D, k_{D}\right)$ is $c_{2}$-bilipschitz with $c_{2}=c_{2}(t, r, s, n)$.

Proof. Let $c_{1}=c_{1}(2 t \sqrt{n} /(r-t), n)$ be the constant given by Lemma 6.5. If $x, y \in \bar{I}^{n}(t)$, we obtain from 6.5 and (6.14):

$$
\begin{aligned}
& k_{D}\left(\alpha_{Q}(x), \alpha_{Q}(y)\right) \leqq \frac{c_{1}\left|\alpha_{Q}(x)-\alpha_{Q}(y)\right|}{d(\bar{Q}(t), \partial D)} \leqq \frac{c_{1}|x-y|}{r-t}, \\
& k_{D}\left(\alpha_{Q}(x), \alpha_{Q}(y)\right) \geqq \frac{\left|\alpha_{Q}(x)-\alpha_{Q}(y)\right|}{c_{1} d(\bar{Q}(t), \partial D)} \geqq \frac{|x-y|}{c_{1}(s-t) \sqrt{n}} .
\end{aligned}
$$

Hence we can choose $c_{2}=\max \left(c_{1} /(r-t), c_{1}(s-t) \sqrt{n}\right)$.

6.16. Notation. Let $f: D \rightarrow D^{\prime}$ be a homeomorphism and let $Q \subset D$ be a cube. We set

$$
d_{Q}^{f}=d\left(f Q, \partial D^{\prime}\right), \quad \beta_{Q}^{f}(z)=\frac{z-f\left(z_{Q}\right)}{d_{Q}^{f}}, \quad f_{Q}=\beta_{Q}^{f} f \alpha_{Q} .
$$

Thus $\beta_{Q}^{f}$ is an affine map of $R^{n}$, and $f_{Q}$ is an embedding defined in $\alpha_{Q}^{-1} D$. If $Q \in \mathscr{K}_{D}(r, s)$, this set always contains the open cube $I^{n}(r)$. Observe that $f_{Q}(0)=0$.

6.17. Theorem. Let $f: D \rightarrow D^{\prime}$ be a homeomorphism. Then the following conditions are equivalent:

(1) $f$ is solid.

(2) There is $r>1$ such that $\left\{f_{Q} \mid I^{n}: Q \in \mathscr{K}_{D}(r)\right\}$ is solid.

(3) If $1 \leqq t<r<s$, then $\left\{f_{Q} \mid I^{n}(t): Q \in \mathscr{K}_{D}(r, s)\right\}$ is solid.

Proof. Trivially (3) implies (2). We show that (2) $\Rightarrow(1) \Rightarrow(3)$.

Suppose that (2) is true. We first show that $f$ is uniformly continuous in the quasihyperbolic metric. Let $\varepsilon>0$. By (2), there is $M>0$ such that $d\left(f_{Q} I^{n}\right) \leqq M$ 
for all $Q \in \mathscr{K}_{D}(r)$. This implies $r_{D^{\prime}}(f Q) \leqq M$. By Lemma 6.5 we obtain

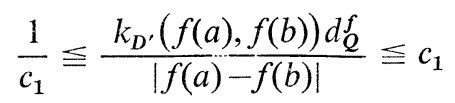

for all $a, b \in Q$, where $c_{1}=c_{1}(M, n)$. By (2), there is $\delta_{1} \in(0,1)$ such that $\left|f_{Q}(x)\right| \leqq$ $\varepsilon / c_{1}$ whenever $|x| \leqq \delta_{1}$ and $Q \in \mathscr{K}_{D}(r)$. Let $c_{2}=c_{2}(1, r, r, n)$ be the constant of Lemma 6.15 , and set $\delta=\delta_{1} / c_{2}$. We show that $k_{D^{\prime}}(f(a), f(b)) \leqq \varepsilon$ whenever $a, b \in D$ and $k_{D}(a, b) \leqq \delta$.

Let $Q \in \mathscr{K}_{D}(r)$ be the cube with center $z_{Q}=a$. By Lemma 6.15, $k_{D}(a, \partial Q) \geqq$ $1 / c_{2} \geqq \delta$, and hence $b \in Q$. Thus $\alpha_{Q}^{-1}(a)=0$ and $\alpha_{Q}^{-1}(b)=b^{\prime} \in I^{n}$. By 6.15, $\left|b^{\prime}\right| \leqq$ $c_{2} k_{D}(a, b) \leqq \delta_{1}$. Hence $\left|f_{Q}\left(b^{\prime}\right)\right| \leqq \varepsilon / c_{1}$. By (6.18) this implies

$$
k_{D^{\prime}}(f(a), f(b)) \leqq c_{1}\left|f_{Q}\left(b^{\prime}\right)\right| \leqq \varepsilon .
$$

Hence $f$ is uniformly continuous.

We next show that $f^{-1}$ is uniformly continuous. Let $\delta^{\prime}>0$. By (2) there is $\varepsilon_{1}>0$ such that $\left|f_{Q}(x)\right| \geqq \varepsilon_{1}$ whenever $Q \in \mathscr{K}_{D}(r), x \in I^{n}$, and $|x| \geqq \min \left(1, \delta^{\prime} / c_{2}\right)$. We show that $k_{D^{\prime}}(f(a), f(b)) \geqq \varepsilon_{1} / c_{1}$ for all $a, b \in D$ such that $k_{D}(a, b) \geqq \delta^{\prime}$.

Choose $Q \in \mathscr{K}_{D}(r)$ such that $z_{Q}=a$. If $b \in Q$, then $\alpha_{Q}^{-1}(b)=b^{\prime} \in I^{n}$, and Lemma 6.15 implies $\left|b^{\prime}\right| \geqq \delta^{\prime} / c_{2}$. Hence $\left|f_{Q}\left(b^{\prime}\right)\right| \geqq \varepsilon_{1}$. By (6.18) this yields

$$
k_{D^{\prime}}(f(a), f(b)) \geqq\left|f_{Q}\left(b^{\prime}\right)\right| / c_{1} \geqq \varepsilon_{1} / c_{1} .
$$

If $b \notin Q$, then (6.18) implies

$$
k_{D^{\prime}}(f(a), f(b)) \geqq k_{D^{\prime}}(f(a), f \partial Q) \geqq d\left(0, f_{Q} \partial I^{n}\right) / c_{1} \geqq \varepsilon_{1} / c_{1} .
$$

Hence $f^{-1}$ is uniformly continuous.

Next suppose that (1) is true. Thus $f$ is $\varphi$-solid for some homeomorphism $\varphi:[0, \infty) \rightarrow[0, \infty)$. We must show that the family $\mathscr{G}=\left\{f_{Q} \mid I^{n}(t): Q \in \mathscr{K}_{D}(r, s)\right\}$ is solid. Since $f_{Q}(0)=0$ and since $I^{n}(t)$ is connected, it suffices to show that $\mathscr{G}$ is equicontinuous and inversely equicontinuous (see 3.8). Let $\varepsilon>0$. For $Q \in \mathscr{K}_{D}(r, s)$ set $Q^{\prime}=\bar{Q}(t)$. Then (6.14) and Lemma 6.9 imply $k_{D}\left(Q^{\prime}\right) \leqq c_{3}$ for some $c_{3}=c_{3}(r, t, n)$. Consequently, $k_{D^{\prime}}\left(f Q^{\prime}\right) \leqq \varphi\left(c_{3}\right)$. By Lemma 6.9 this yields $r_{D^{\prime}}\left(f Q^{\prime}\right) \leqq 2\left(e^{\varphi\left(c_{3}\right)}-1\right)=$ $c_{4}$. Hence, by Lemma 6.5,

$$
\frac{1}{c_{1}^{\prime}} \leqq \frac{k_{D^{\prime}}(f(a), f(b)) d_{Q^{\prime}}^{f}}{|f(a)-f(b)|} \leqq c_{1}^{\prime}
$$

for all $a, b \in Q^{\prime}$, where $c_{1}^{\prime}=c_{1}\left(c_{4}, n\right)$.

Let $x, y \in I^{n}(t)$. Since $d_{Q^{\prime}}^{f} \leqq d_{Q}^{f},(6.19)$ and 6.15 imply

$$
\begin{aligned}
\left|f_{Q}(x)-f_{Q}(y)\right| & \leqq c_{1}^{\prime} k_{D^{\prime}}\left(f\left(\alpha_{Q}(x)\right), f\left(\alpha_{Q}(y)\right)\right) \\
& \leqq c_{1}^{\prime} \varphi\left(k_{D}\left(\alpha_{Q}(x), \alpha_{Q}(y)\right)\right) \\
& \leqq c_{1}^{\prime} \varphi\left(c_{2}|x-y|\right),
\end{aligned}
$$

where $c_{2}=c_{2}(t, r, s, n)$. Hence $\mathscr{G}$ is equicontinuous. 
We finally show that $\mathscr{G}$ is inversely equicontinuous. Let $\delta>0$, and let $x, y \in I^{n}(t)$ with $|x-y| \geqq \delta$. We want to find a positive lower bound for $\left|f_{Q}(x)-f_{Q}(y)\right|$, valid for all $Q \in \mathscr{K}_{D}(r, s)$. By (6.19) and 6.15 we obtain

$$
\begin{aligned}
\left|f_{Q}(x)-f_{Q}(y)\right| & \equiv k_{D^{\prime}}\left(f\left(\alpha_{Q}(x)\right), f\left(\alpha_{Q}(y)\right)\right) d_{Q^{\prime}}^{f} / c_{1}^{\prime} d_{Q}^{f} \\
& \geqq \varphi^{-1}\left(\delta / c_{2}\right) d_{Q^{\prime}}^{f} / c_{1}^{\prime} d_{Q}^{f} .
\end{aligned}
$$

Hence it suffices to find a positive lower bound for $d_{Q^{\prime}}^{f} / d_{Q}^{f}$. First, we have $d_{Q^{\prime}}^{f} / d_{Q}^{f} \geqq r_{D^{\prime}}(f Q) / r_{D^{\prime}}\left(f Q^{\prime}\right)$. Here $r_{D^{\prime}}\left(f Q^{\prime}\right) \leqq c_{4}$. Hence, if $r_{D^{\prime}}(f Q) \geqq 1, d_{Q^{\prime}}^{f} / d_{Q}^{f} \geqq 1 / c_{4}$. Suppose that $r_{D^{\prime}}(f Q)<1$. Then Lemma 6.9 implies

$$
r_{D^{\prime}}(f Q) \geqq k_{D^{\prime}}(f Q) / c_{1}(1, n) \geqq \varphi^{-1}\left(k_{D}(Q)\right) / c_{1}(1, n),
$$

where, by Lemma 6.9 and by (6.14),

$$
k_{D}(Q) \geqq \log \left(1+r_{D}(Q) / 2\right) \geqq \log (1+1 /(s-1)) .
$$

The theorem is proved.

6.20. Remark. The proof of Theorem 6.17 shows that a quantitative version is also true. Thus, if $f: D \rightarrow D^{\prime}$ is a $\varphi$-solid homeomorphism and if $1 \leqq t<r<s$, then $\left\{f_{Q} \mid I^{n}(t): Q \in \mathscr{K}_{D}(r, s)\right\}$ is contained in a solid family $\mathscr{F}$ of embeddings $I^{n}(t) \rightarrow R^{n}$, depending only on $\varphi, t, r, s$ and $n$. Conversely, if $\mathscr{F}$ is a solid family of embeddings of $I^{n}$ and if $\left\{f_{Q} \mid I^{n}: Q \in \mathscr{K}_{D}(r)\right\} \subset \mathscr{F}$, then $f$ is $\varphi$-solid with $\varphi$ depending only on $\mathscr{F}$ and $r$.

6.21. Lemma. Suppose that a homeomorphism $f: D \rightarrow D^{\prime}$ is locally L-bilipschitz in the quasihyperbolic metric. Then $f$ is L-bilipschitz in the quasihyperbolic metric.

Proof. It suffices to show that $f$ is $L$-lipschitz. Let $a, b \in D$, and choose a quasihyperbolic geodesic joining $a$ and $b$. Divide $\alpha$ to subpaths $\alpha_{1}, \ldots, \alpha_{s}$ such that $f$ is $L$-bilipschitz on each $\operatorname{im} \alpha_{j}$. Let $a_{j-1}$ and $a_{j}$ be the end points of $\alpha_{j}$. Then

$$
\begin{aligned}
k_{D^{\prime}}(f(a), f(b)) & \leqq \sum_{j=1}^{s} k_{D^{\prime}}\left(f\left(a_{j-1}\right), f\left(a_{j}\right)\right) \\
& \leqq L \sum_{j=1}^{s} k_{D}\left(a_{j-1}, a_{j}\right) \\
& =L k_{D}(a, b) .
\end{aligned}
$$

\section{Lipschitz approximation of solid and QC homeomorphisms}

7.1. In this section we first show that if $n \neq 4$, every solid homeomorphism $D \rightarrow D^{\prime}$ can be approximated arbitrarily closely in the quasihyperbolic metric by homeomorphisms which are bilipschitz in the quasihyperbolic metrics of $D$ and $D^{\prime}$ (and hence QC). The method is a variation of the proof of the extension theorem 
in $\left[\mathrm{TV}_{2}\right]$. In fact, in $\left[\mathrm{TV}_{2}\right]$ we first extended a QC homeomorphism $f: R^{n} \rightarrow R^{n}$ to a homeomorphism $F_{f}: R_{+}^{n+1} \rightarrow R_{+}^{n+1}$ by

$$
F_{f}(x, t)=\left(f(x), \max \left\{|f(y)-f(x)|: y \in \bar{B}^{n}(x, t)\right\}\right) .
$$

Then the restriction of $F_{f}$ to $H=\operatorname{int} R_{+}^{n+1}$ is solid but not necessarily QC. We constructed an approximation $F$ of $F_{f} \mid H$ which is bilipschitz in the hyperbolic metric and hence QC. Since $k_{H}\left(F, F_{f}\right)<\infty, F$ and $F_{f}$ have the same boundary values, and thus $F \cup f$ is the desired extension of $f$.

The proof in $\left[\mathrm{TV}_{2}\right]$ was based on the qualitative part of Lemma 3.9 of the present paper. However, it is possible to simplify the proof by making also use of the quantitative part of 3.9. Indeed, it is not necessary to introduce the points $z^{\prime \prime}$ in $\left[\mathrm{TV}_{2}\right.$, (2.8)], and Carleson's finiteness idea is only needed in the approximation of the maps $F_{f} \mid Q$, not in the glueing process. We shall use this simplification in the proof of Theorems 7.4 and 7.18.

As an application of our result we extend to higher dimensions a result of Ahlfors concerning QC reflections in $R^{2}$.

In general, the dilatation of the approximating homeomorphism depends on the closeness of the approximation. However, we show in 7.18 that every $K$-QC homeomorphism $D \rightarrow D^{\prime}, n \neq 4$, can be approximated arbitrarily closely in the majorant topology by LIP homeomorphisms which are $K_{1}$-QC for some $K_{1}=$ $K_{1}(K, n)$.

7.2. A cube decomposition. Let $D$ be a proper subdomain of $R^{n}$. We define a decomposition $\mathscr{K}(D)$ of $D$ into closed cubes as follows. For $k \in \mathbf{Z}$ let $\mathscr{L}(k)$ be the decomposition of $R^{n}$ into closed $n$-cubes of side length $2^{k}$ and with vertices in $2^{k} \mathbf{Z}^{n}$. Set $\mathscr{L}=\cup\{\mathscr{L}(k): k \in \mathbf{Z}\}$ and $\mathscr{K}^{0}(D)=\{Q \in \mathscr{L}: \bar{Q}(3) \subset D\}$ (see 6.13 for notation). Each cube of $\mathscr{K}^{0}(D)$ is contained in a maximal cube of $\mathscr{K}^{0}(D)$. Let $\mathscr{K}^{1}(D)$ be the family of all maximal cubes in $\mathscr{K}^{0}(D)$. We divide each cube of $\mathscr{K}^{1}(D)$ into $N=2^{n}$ cubes bisecting the sides of $Q$. This gives the family $\mathscr{K}(D)$ of cubes in $D$.

We divide $\mathscr{K}(D)$ into disjoint subfamilies $\mathscr{K}_{1}(D), \ldots, \mathscr{K}_{N}(D)$ as follows: Divide $I^{n}$ into cubes $Q_{1}, \ldots, Q_{N}$ bisecting the sides. Then set $\mathscr{K}_{j}(D)=\left\{\alpha_{Q} Q_{j}: Q \in \mathscr{K}^{1}(D)\right\}$ where $\alpha_{Q}$ is as in 6.13 .

Similar decompositions have been used, for example, by H. Whitney [Wh, p. 67], E. Stein [St, p. 167] and M. Kiikka [Kk, p. 7]. The following properties of $\mathscr{K}(D)$ are readily verified (notation as in 6.13 ):

7.3. Lemma. (1) $\cup \mathscr{K}(D)=D$.

(2) If $Q, R \in \mathscr{K}(D), Q \neq R$ and $Q \cap R \neq \emptyset$, then int $Q \cap \operatorname{int} R=\emptyset$ and $\lambda_{Q} / \lambda_{R} \in\{1 / 2,1,2\}$.

(3) $\mathscr{K}(D) \subset \mathscr{K}_{D}(5,15)$.

(4) If $Q \in \mathscr{K}(D)$, then $1 / 7 \leqq r_{D}(Q) \leqq \sqrt{n} / 2$.

(5) If $Q, R \in \mathscr{K}_{i}(D)$, then $Q \cap R=\emptyset$. 
7.4. Theorem. Let $n \neq 4$, let $\varepsilon>0$, and let $\varphi:[0, \infty) \rightarrow[0, \infty)$ be a homeomorphism. Then there is a number $L=L(\varphi, \varepsilon, n) \geqq 1$ with the following property:

Suppose that $D$ and $D^{\prime}$ are proper subciomains of $R^{n}$ and that $f: D \rightarrow D^{\prime}$ is a $\varphi$-solid homeomorphism. Then there is a homeomorphism $F: D \rightarrow D^{\prime}$ such that

(1) $k_{D^{\prime}}(F, f) \leqq \varepsilon$,

(2) $F$ is L-bilipschitz in the quasihyperbolic metrics of $D$ and $D^{\prime}$,

(3) $F$ is $L^{2 n-2}$-QC.

Proof. The proof of Theorem 7.4 will be completed in 7.11. From now on, we assume that $n, \varepsilon, \varphi$ are given as in the theorem and that $f: D \rightarrow D^{\prime}$ is $\varphi$-solid.

7.5. Lemma. There is $M=M(\varphi, n)$ such that

$$
1 / M \leqq d_{Q}^{f} / d_{R}^{f} \leqq M, \quad d_{Q}^{f} \leqq M d\left(f \bar{Q}(2), \partial D^{\prime}\right)
$$

whenever $Q, R \in \mathscr{K}(D)$ with $Q \cap R \neq \emptyset$.

Proof. By 6.9 and 7.3.(4), $k_{D}(Q) \leqq M_{1}$ for some $M_{1}=M_{1}(n)$. Hence $k_{D^{\prime}}(f Q) \leqq$ $\varphi\left(M_{1}\right)$. Since $d_{R}^{f} \leqq d_{Q}^{f}+d(f Q)=\left(1+r_{D^{\prime}}(f Q)\right) d_{Q}^{f}$, Lemma 6.9 implies $d_{R}^{f} \leqq M d_{Q}^{f}$ with $M=2 e^{\varphi\left(M_{1}\right)}$. By symmetry, $d_{Q}^{f} \leqq M d_{R}^{f}$. The last inequality follows from the fact that $\bar{Q}(2) \subset \cup\{R \in \mathscr{K}(D): R \cap Q \neq \emptyset\}$.

7.6. Lemma. There is a number $c_{3}=c_{3}(\varphi, n) \geqq 1$ such that

$$
\beta_{Q}^{f} \mid f \bar{Q}(2):\left(f \bar{Q}(2), k_{D^{\prime}}\right) \rightarrow\left(R^{n}, d\right)
$$

is $c_{3}$-bilipschitz for every $Q \in \mathscr{K}(D)$.

Proof. Set $A=f \bar{Q}(2)$. Since $\bar{Q}(2) \in \mathscr{K}_{D}(5 / 2,15 / 2), 6.9$ and (6.14) imply $r_{D^{\prime}}(A) \leqq M_{2}=M_{2}(\varphi, n)$. Hence we can apply Lemma 6.5 with $c_{1}=c_{1}\left(M_{2}, n\right)$. For $a, b \in A$ we obtain

$$
\left|\beta_{Q}^{f}(a)-\beta_{Q}^{f}(b)\right|=|a-b| / d_{Q}^{f} \leqq c_{1} k_{D^{\prime}}(a, b) d\left(A, \partial D^{\prime}\right) / d_{Q}^{f} \leqq c_{1} k_{D^{\prime}}(a, b) .
$$

By 7.5 we also get

$$
\left|\beta_{Q}^{f}(a)-\beta_{Q}^{f}(b)\right| \geqq k_{D^{\prime}}(a, b) d\left(A, \partial D^{\prime}\right) / c_{1} d_{Q}^{f} \geqq k_{D^{\prime}}(a, b) / M c_{1} .
$$

Hence the lemma is true with $c_{3}=M c_{1}$.

7.7. Constructions. We set

$$
\begin{aligned}
\mathscr{K}_{i}^{*}(D) & =\mathscr{K}_{1}(D) \cup \ldots \cup \mathscr{K}_{i}(D), \\
V_{i}(D) & =\cup\left\{Q\left(1+2^{-i-1}\right): Q \in \mathscr{K}_{i}^{*}(D)\right\}, \\
W_{i}(D) & =\cup\left\{Q\left(1+2^{-i-2}\right): Q \in \mathscr{K}_{i}^{*}(D)\right\}
\end{aligned}
$$

with $V_{0}(D)=W_{0}(D)=\emptyset$. If $Q \in \mathscr{K}_{i}(D)$ and $1<t \leqq 3 / 2$, we set

$$
\begin{aligned}
V_{Q}(D, t) & =I^{n}(t) \cap \alpha_{Q}^{-1} V_{i-1}(D), \\
W_{Q}(D, t) & =I^{n}(t) \cap \alpha_{Q}^{-1} W_{i-1}(D) .
\end{aligned}
$$


Then for every $t \in(1,3 / 2]$ there is a finite family $S(n, t)$ such that $V_{Q}(D, t)$ and $W_{Q}(D, t)$ belong to $S(n, t)$ for every dcmain $D$ and for every $Q \in \mathscr{K}(D)$.

By 6.17, 6.10 and 7.3.(3), there is a solid family $\mathscr{G}=\mathscr{G}(\varphi, n) \subset E\left(I^{n}(2) ; R^{n}\right)$ such that $f_{Q} \mid I^{n}(2) \in \mathscr{G}$ for every $\varphi$-solid homeomorphism $f: D \rightarrow D^{\prime}$ and for every $Q \in \mathscr{K}(D)$.

We apply Lemma 3.9 with $U=I^{n}(3 / 2), \quad U^{\prime}=I^{n}(4 / 3), \quad V=V_{Q}(D, 3 / 2), \quad W=$ $W_{Q}(D, 3 / 2)$ for $Q \in \mathscr{K}(D)$, and with $\mathscr{F}=\left\{g \mid I^{n}(3 / 2): g \in \mathscr{G}\right\}$. Since the family $S(n, 3 / 2)$ is finite, we obtain:

7.8. Lemma. Let $\varepsilon^{\prime}>0$ and $L \geqq 1$. Then there are positive numbers $\delta=\delta\left(\varepsilon^{\prime}, \varphi, n\right) \leqq \varepsilon^{\prime}$ and $L^{\prime}=L^{\prime}\left(\varepsilon^{\prime}, \varphi, n, L\right) \geqq L$ with the following properties:

Let $D$ be a proper subdomain of $R^{n}$, let $Q \in \mathscr{K}(D)$, let $h: V_{Q}(D, 3 / 2) \rightarrow R^{n}$ be a locally L-bilipschitz embedding, and let $g \in \mathscr{F}$ be such that $d\left(g, h ; V_{Q}(D, 3 / 2)\right) \leqq \delta$. Then there is a LIP embedding $h^{\prime}: I^{n}(3 / 2) \rightarrow R^{n}$ such that

(1) $d\left(h^{\prime}, g ; I^{n}(4 / 3)\right) \leqq \varepsilon^{\prime}$,

(2) $h^{\prime}=h$ in $W_{Q}(D, 4 / 3)$,

(3) $h^{\prime} \mid I^{n}(4 / 3)$ is $L^{\prime}$-bilipschitz.

7.9. Constructions. Since $\mathscr{G}$ is solid, there is a number $q=q(\varphi, n)>0$ such that $|g(x)-g(y)| \geqq q$ whenever $g \in \mathscr{G}$ and $x, y \in I^{n}(7 / 4)$ with $|x-y| \geqq 1 / 8$. Let $c_{2}=c_{2}(3 / 2,5,15, n), c_{3}=c_{3}(\varphi, n)$ and $M=M(\varphi, n)$ be as in 6.15, 7.6 and 7.5. Define numbers $\delta_{N} \geqq \delta_{N-1} \geqq \ldots \geqq \delta_{0}>0$ by $\delta_{N}=\min \left(q /(M+2), \varepsilon / c_{3}\right)$ and $\delta_{j-1}=$ $\delta\left(\delta_{j}, \varphi, n\right) / M$ where $\delta()$ is as in 7.8. We also define numbers $L_{0} \leqq \ldots \leqq L_{N}$ by $L_{0}=1$ and $L_{j}=c_{2} c_{3} L^{\prime}\left(\delta_{j}, \varphi, n, c_{2} c_{3} L_{j-1}\right)$, where $L^{\prime}()$ is as in 7.8. Observe that the sequences $\left(\delta_{0}, \ldots, \delta_{N}\right)$ and $\left(L_{0}, \ldots, L_{N}\right)$ deperd only on $\varphi, n$ and $\varepsilon$. We show by induction that the following lemma is true for every integer $j \in[0, N]$ :

$7.10_{j}$. Lemma. There is an embedding $F_{j}: V_{j}(D) \rightarrow D^{\prime}$ with the following properties:

(1) $d\left(F_{j}, f ; Q\left(1+2^{-j-1}\right)\right) \leqq \delta_{j} d_{Q}^{f}$ for every $Q \in \mathscr{K}_{j}^{*}(D)$.

(2) $F_{j} Q\left(1+2^{-j-1}\right) \subset f Q(3 / 2)$ for every $Q \in \mathscr{K}_{j}^{*}(D)$.

(3) $F_{j}$ is locally $L_{j}$-bilipschitz in the quasihyperbolic metrics of $D$ and $D^{\prime}$.

Proof. Since $V_{0}(D)=\emptyset, 7.10_{0}$ is true. Suppose that $7.10_{j-1}$ is true. Thus we have an embedding $F_{j-1}: V_{j-1}(D) \rightarrow D^{\prime}$. We define $F_{j}(x)=F_{j-1}(x)$ for $x \in W_{j-1}(D)$. Let $Q \in \mathscr{K}_{j}(D)$. Then $f_{Q} \mid I^{n}(3 / 2) \in \mathscr{F}$. Set $h_{Q}=\beta_{Q}^{f} F_{j-1} \alpha_{Q} \mid V_{Q}(D, 3 / 2)$. We first show that $d\left(h_{Q}, f_{Q} ; V_{Q}(D, 3 / 2)\right) \leqq \delta\left(\delta_{j}, \varphi, n\right)$. Let $x \in V_{Q}(D, 3 / 2)$. Then $x \in \alpha_{Q}^{-1} R\left(1+2^{-j}\right)$ for some $R \in \mathscr{K}_{j-1}^{*}(D)$ with $R \cap Q \neq \emptyset$. By 7.5 and $7.10_{j-1}$ we obtain

$$
\begin{aligned}
\left|h_{Q}(x)-f_{Q}(x)\right| & =\left|f\left(\alpha_{Q}(x)\right)-F_{j-1}\left(\alpha_{Q}(x)\right)\right| / d_{Q}^{f} \\
& \leqq M \delta_{j-1}=\delta\left(\delta_{j}, \varphi, n\right) .
\end{aligned}
$$

Hence we can apply Lemma 7.8 with $g=f_{Q}, h=h_{Q}$ and $\varepsilon^{\prime}=\delta_{j}$. We obtain a LIP embedding $h_{Q}^{\prime}: I^{n}(3 / 2) \rightarrow R^{n}$ such that (a) $d\left(h_{Q}^{\prime}, f_{Q} ; I^{n}(4 / 3)\right) \leqq \delta_{j}$ and (b) $h_{Q}^{\prime}=h_{\boldsymbol{Q}}$ 
in $W_{Q}(D, 4 / 3)$. By 6.15 and $7.6, h_{Q}$ is locally $c_{2} c_{3} L_{j-1}$-bilipschitz in the euclidean metric. Hence $h_{Q}^{\prime} \mid I^{n}(4 / 3)$ is $L_{j} / c_{2} c_{3}$-bilipschitz. Setting $F_{j}=\left(\beta_{Q}^{f}\right)^{-1} h_{Q}^{\prime} \alpha_{Q}^{-1}$ in $Q\left(1+2^{-j-1}\right)$ we obtain a well-defined map $F_{j}: V_{j}(D) \rightarrow R^{n}$. We show that $F_{j}$ satisfies the conditions (1), (2), (3) and that $F_{j}$ is injective.

Let $Q \in \mathscr{K}_{j}^{*}(D)$. If $Q \in \mathscr{K}_{j-1}^{*}(D)$, (1) follows from $7.10_{j-1}$. If $Q \in \mathscr{K}_{j}(D)$, we obtain

$$
d\left(F_{j}, f ; Q\left(1+2^{-j-1}\right)\right)=d_{Q}^{f} d\left(h_{Q}^{\prime}, f_{Q} ; I^{n}\left(1+2^{-j-1}\right)\right) \leqq \delta_{j} d_{Q}^{f} .
$$

To prove (2), let again $Q \in \mathscr{K}_{j}^{*}(D)$. If $Q \in \mathscr{K}_{j-1}^{*}(D)$, (2) follows by induction. Suppose that $Q \in \mathscr{K}_{j}(D)$. Then $d\left(h_{Q}^{\prime}, f_{Q} ; I^{n}(4 / 3)\right) \leqq \delta_{j} \leqq \delta_{N}<q$. By the choice of $q$, this implies $h_{Q}^{\prime} I^{n}\left(1+2^{-j-1}\right) \subset f_{Q} I^{n}(3 / 2)$. Hence (2) is true. Observe that (2) implies im $F_{j} \subset D^{\prime}$.

If $Q \in \mathscr{K}_{j-1}^{*}(D), \quad F_{j}$ is locally $L_{j}$-bilipschitz in $Q\left(1+2^{-j-1}\right)$ by induction: If $Q \in \mathscr{K}_{j}(D)$, then $6.15,7.6$ and (2) imply that $F_{j} \mid Q\left(1+2^{-j-1}\right)$ is $L_{j}$-bilipschitz. Hence $F_{j}$ is a locally $L_{j}$-bilipschitz immersion. We finally show that $F_{j}$ is injective. We know that $F_{j} \mid Q\left(1+2^{-j-1}\right)$ is injective for every $Q \in \mathscr{K}_{j}^{*}(D)$. Moreover, if $Q, R \in \mathscr{K}_{j}^{*}(D)$ and $Q \cap R=\emptyset$, then (2) implies that

$$
F_{j} Q\left(1+2^{-j-1}\right) \cap F_{j} R\left(1+2^{-j-1}\right)=\emptyset .
$$

Hence it suffices to show that $F_{j}(x) \neq F_{j}(y)$ whenever $j \geqq 2, x \neq y, x \in Q\left(1+2^{-j-1}\right)$ and $y \in R\left(1+2^{-j-1}\right)$ where $Q \in \mathscr{K}_{j}(D), R \in \mathscr{K}_{j-1}^{*}(D)$ and $Q \cap R \neq \emptyset$. The equality $F_{j}=\left(\beta_{Q}^{f}\right)^{-1} h_{Q}^{\prime} \alpha_{Q}^{-1}$ is valid in $Q(4 / 3) \cap V_{j}(D)$. Hence we may assume that $y \notin Q(4 / 3)$. By the choice of $q$, we have $d\left(f_{Q}\left(\alpha_{Q}^{-1}(x)\right), f_{Q} \partial I^{n}(5 / 4)\right) \geqq q$. Hence $|f(x)-f(y)| \geqq q d_{Q}^{f}$. By (1) and 7.5 we obtain

$$
\begin{aligned}
\left|F_{j}(x)-F_{j}(y)\right| & \geqq|f(x)-f(y)|-\left|F_{j}(x)-f(x)\right|-\left|F_{j}(y)-f(y)\right| \\
& \geqq q d_{Q}^{f}-\delta_{j} d_{Q}^{f}-\delta_{j} d_{R}^{f} \\
& \geqq d_{Q}^{f}\left(q-(M+1) \delta_{N}\right) \geqq d_{Q}^{f} \delta_{N}>0 . \quad \square
\end{aligned}
$$

7.11. Completion of the proof of Theorem 7.4. We show that the theorem is true with $L=L_{N}$, defined in 7.9. Let $f: D \rightarrow D^{\prime}$ be $\varphi$-solid. We show that the map $F_{N}$ of $7.10_{N}$ is the required $F$. First, $F_{N}$ is an embedding of $D$ into $D^{\prime}$, and $F_{N}$ is locally $L_{N}$-bilipschitz in the quasihyperbolic metric. To prove the condition (1) of Theorem 7.4, let $x \in D$. Choose $Q \in \mathscr{K}(D)$ containing $x$. Then 7.10. (1) yields

$$
\left|F_{N}(x)-f(x)\right| \leqq \delta_{N} d_{Q}^{f} \leqq \varepsilon d_{Q}^{f} / c_{3} .
$$

By 7.6 and 7.10. (2), this implies $k_{D^{\prime}}\left(F_{N}(x), f(x)\right) \leqq \varepsilon$. Hence (1) is true. Moreover, (1) implies that $F_{N}$ is a homeomorphism of $D$ onto $D^{\prime}$.

We already proved that $F_{N}$ is locally $L_{N}$-bilipschitz in the quasihyperbolic metric. By $6.21, F_{N}$ is $L_{N}$-bilipschitz.

To prove that $F_{N}$ is $L_{N}^{2 n-2}$-QC, it suffices to show that the linear dilatation $H\left(x, F_{N}\right)$ is at most $L_{N}^{2}\left[V \ddot{a}_{1}, 34.2\right]$. This follows from (2) and from Remark 6.8. 
7.12. Corollary. Let $n \neq 4$, let $\varepsilon>0$ and let $K \geqq 1$. Then there is $L=$ $L(K, \varepsilon, n) \geqq 1$ such that if $D \subset R^{n}$ is a proper subdomain and if $f: D \rightarrow D^{\prime}$ is a $K$-QC homeomorphism, then there is a homeomorphism $F: D \rightarrow D^{\prime}$ such that

(1) $k_{D^{\prime}}(F, f) \leqq \varepsilon$,

(2) $F$ is $L$-bilipschitz in the quasihyperbolic metrics of $D$ and $D^{\prime}$,

(3) $F$ is $L^{2 n-2}$-QC.

7.13. Remark. It follows from Theorem 7.4 that solid homeomorphisms and QC homeomorphisms have similar boundary properties, at least for $n \neq 4$. For example, if $b$ is an isolated boundary point of $D$ and if $f: D \rightarrow D^{\prime}$ is solid, then $f$ has a limit $b^{\prime}$ at $b$, and $b^{\prime}$ is an isolated boundary point of $D^{\prime}$. Furthermore, let $H^{n}=\operatorname{int} R_{+}^{n}$, and let $f: H^{n} \rightarrow H^{n}$ be solid. The $f$ can be extended to a homeomorphism $g: \bar{R}_{+}^{n} \rightarrow \bar{R}_{+}^{n}$, and the induced boundary map $g \mid \bar{R}^{n-1}$ is QC. It is possible to give direct proofs for these and several other results on solid homeomorphisms. These proofs are also valid for $n=4$. Cf. [Th, 5.9.6].

7.14. Quasiconformal reflections. Let $E$ be a closed connected set in $R^{n}$ such that $R^{n} \backslash E$ has exactly two components $D_{1}$ and $D_{2}$. A homeomorphism $f: R^{n} \rightarrow R^{n}$ is said to be a reflection in $E$ if $f \mid E=$ id and $f D_{1}=D_{2}$. Then $f D_{2}=D_{1}, f$ is sensereversing, and $\partial D_{1}=E=\partial D_{2}$. If $f$ is a reflection in $E$, we can always find a reflection $g$ in $E$ which is also an involution, that is, $g g=\mathrm{id}$. For example, we can choose $g=\left(f \mid \bar{D}_{1}\right) \cup\left(f^{-1} \mid D_{2}\right)$.

L. V. Ahlfors [Ah, p. 80] proved that if $f: R^{2} \rightarrow R^{2}$ is a QC reflection in $E$, there is a reflection $F$ in $E$ which is bilipschitz in the euclidean metric. We shall extend this result for all dimensions $n \neq 4$.

On the other hand, Ahlfors [Ah, p. 75] also proved that if $E$ admits a QC reflection, $E \cup \infty$ is a QC circle. (By a classical result of L. E. J. Brouwer, it is always a topological circle.) This result cannot be extended to higher dimensions. Indeed, for every $n \geqq 3$ there is a QC reflection $f: R^{n} \rightarrow R^{n}$ in a set $E$ such that $E \cup \infty$ is a topological sphere but no nonempty open subset of $E$ can be quasisymmetrically embedded into $R^{n-1}$. See $\left[\mathrm{Tu}_{2}\right.$, Example 2].

7.15. Theorem. Suppose that $n \neq 4$ and that $f: R^{n} \rightarrow R^{n}$ is a $K$-QC reflection in $E \subset R^{n}$. Then there is a reflection $F: R^{n} \rightarrow R^{n}$ in $E$ such that $F$ is L-bilipschitz in the euclidean metric with $L$ depending only on $K$ and $n$.

Proof. Let $D_{1}, D_{2}$ be the components of $R^{n} \backslash E$, and let $f_{1}: D_{1} \rightarrow D_{2}$ and $f_{2}: D_{2} \rightarrow D_{1}$ be the $K$-QC (sense-reversing) homeomorphisms defined by $f$. Applying 7.12 with $\varepsilon=1$ we find homeomorphisms $F_{1}: D_{1} \rightarrow D_{2}$ and $F_{2}: D_{2} \rightarrow D_{1}$ such that $k_{D_{2}}\left(F_{1}, f_{1} ; D_{1}\right) \leqq 1, k_{D_{1}}\left(F_{2}, f_{2} ; D_{2}\right) \leqq 1$, and such that $F_{1}$ and $F_{2}$ are $L_{1}$-bilipschitz in the quasihyperbolic metrics of $D_{1}$ and $D_{2}$ with $L_{1}=L_{1}(K, n)$. Then $F=F_{1} \cup$ $F_{2} \cup($ id $\mid E)$ is a reflection in $E$. 
For $x \in R^{n}$ and $r>0$ we let as usual $L(x, f, r)$ and $l(x, f, r)$ denote the maximum and minimum of $|f(y)-f(x)|$ over $y \in S^{n-1}(x, r)$. Then there is $H=$ $H(K, n)$ such that $L(x, f, r) \leqq H l(x, f, r)$; see, for example, the proof of [Vä 22.3]. We show that $F$ is $H e L_{1}$-bilipschitz, $e=2.7182 \ldots$, in the euclidean metric. By Lemma 2.2, it suffices to show that

$$
L(x, F) \leqq H e L_{1}, \quad L\left(x, F^{-1}\right) \leqq H e L_{1}
$$

for all $x \in R^{n}$.

Let first $x \in R^{n} \backslash E$, say $x \in D_{1}$. Set $r=d(x, E), r^{\prime}=d(f(x), E), r^{\prime \prime}=d(F(x), E)$. Choose $y$ and $z$ in $E$ such that $|y-x|=r$ and $|z-f(x)|=r^{\prime}$. Setting $s=|x-z|$ we obtain

$$
\begin{aligned}
& r^{\prime} \leqq|f(x)-y| \leqq L(y, f, r) \leqq H l(y, f, r) \leqq H r, \\
& r^{\prime}=|f(x)-z| \geqq l(z, f, s) \geqq L(z, f, s) / H \geqq s / H \geqq r / H .
\end{aligned}
$$

Furthermore, since $k_{D_{2}}(F(x), f(x)) \leqq 1$, [GP, (2.1)] implies

$$
r^{\prime} / e \leqq r^{\prime \prime} \leqq e r^{\prime},
$$

and (7.16) follows by Lemma 6.5.

Next assume $x \in E$. Let $r>0$ and let $|y-x|=r$. If $y \in D_{1},|f(y)-x| \leqq$ $H l(x, f, r) \leqq H r$. Since $k_{D_{2}}(F(y), f(y)) \leqq 1, \quad[\mathrm{GP},(2.2)]$ implies $|F(y)-f(y)| \leqq$ $(e-1) d(f(y), E) \leqq(e-1)|f(y)-x| \leqq(e-1) H r$. Hence $|F(y)-x| \leqq e H r$, which implies the first inequality of (7.16).

Similarly, $|f(y)-x| \geqq L(x, f, r) / H \geqq r / H$, and $|F(y)-f(y)| \leqq(e-1) d(F(y), E) \leqq$ $(e-1)|F(y)-x|$, which implies $|F(y)-x| \geqq|f(y)-x| / e \geqq r / H e$, and we obtain the second inequality of (7.16).

7.17. Approximation by LIP homeomorphisms. In Theorem 7.4 we approximated a solid homeomorphism $f: D \rightarrow D^{\prime}$ by a homeomorphism $F$ which was bilipschitz in the quasihyperbolic metric. We shall next give a related result which is weaker in two respects: (1) $f$ is supposed to be $K$-QC and not only solid, (2) $F$ is only locally bilipschitz. On the other hand, it is stronger in the following respects: $F$ is $K_{1}$-QC with $K_{1}=K_{1}(K, n)$, (2) the approximation is in the majorant topology and not in the uniform topology of the quasihyperbolic metric, (3) $D$ can be the whole space $R^{n}$, and (4) it is strongly relative. Since the proof is a modification of the proof of Theorem 7.4, we shall omit some details. The case $n \leqq 3$ was proved by M. Kiikka [Kk] who showed that in this case $F$ can be chosen to be PL.

7.18. Theorem. Let $D$ be a domain in $R^{n}, n \neq 4$, let $U$ be an open set in $D$, and let $\varepsilon: U \rightarrow R^{1}$ be continuous and positive. Then for every $K$-QChomeomorphism $f: D \rightarrow D^{\prime}$ there is a homeomorphism $F: D \rightarrow D^{\prime}$ such that

(1) $|F(x)-f(x)| \leqq \varepsilon(x)$ for every $x \in U$,

(2) $F=f$ in $D \backslash U$,

(3) $F \mid U$ is a LIP embedding,

(4) $F$ is $K_{1}$-QC with $K_{1}$ depending only on $K$ and $n$. 
Proof. The theorem reduces easily to the absolute case $U=D$. Indeed, in the general case we first replace $\varepsilon(x)$ by $\min (\varepsilon(x), d(x, \partial U))$ and then apply the special case in each component of $U$. We obtain a $K_{1}$-QC LIP homeomorphism $F_{1}: U \rightarrow f U$ which can be extended by $f \mid D \backslash U$ to a homeomorphism $F: D \rightarrow D^{\prime}$, which is $K_{2}-\mathrm{QC}$ with $K_{2}=\max \left(K, K_{1}\right)$ [Vä ${ }_{2}$, Theorem 2].

From now on, we suppose that $U=D$ and that $f: D \rightarrow D^{\prime}$ is a $K$-QC homeomorphism. We may assume that $\varepsilon(x) \leqq d\left(f(x), \partial D^{\prime}\right)$ if $D^{\prime} \neq R^{n}$ and that $\varepsilon(x) \leqq$ $(1+|f(x)|)^{-1}$. We replace the cube decomposition $\mathscr{K}(D)$ of 7.2 by another decomposition $\mathscr{H}(D)$ which also depends on $f$ and $\varepsilon$. Let $\mathscr{L}$ be as in 7.2 , and let $\mathscr{H}^{1}(D)$ be the family of all maximal cubes of $\mathscr{L}$ satisfying the conditions $\bar{Q}(3) \subset D$ and $d(f \bar{Q}(3)) \leqq \min \varepsilon \bar{Q}(3)$. Dividing each cube of $\mathscr{H}^{1}(D)$ into $N=2^{n}$ subcubes by bisecting the sides we obtain the family $\mathscr{H}(D)$. It has the following properties:

(1) $\cup \mathscr{H}(D)=D$.

(2) If $Q, R \in \mathscr{H}(D), Q \neq R$ and $Q \cap R \neq \emptyset$, then int $Q \cap$ int $R=\emptyset$, and $\lambda_{Q} / \lambda_{R} \in\{1 / 2,1,2\}$.

(3) If $Q \in \mathscr{H}(D), \bar{Q}(5) \subset D$.

(4) If $x \in Q \in \mathscr{H}(D), d(f Q) \leqq \varepsilon(x)$.

We divide $\mathscr{H}(D)$ into subfamilies $\mathscr{H}_{1}(D), \ldots, \mathscr{H}_{N}(D)$ as in 7.2 , and set $\mathscr{H}_{j}^{*}(D)=$ $\mathscr{H}_{1}(D) \cup \ldots \cup \mathscr{H}_{j}(D)$.

We next show that there is $M=M(K, n)$ such that

$$
\frac{1}{M} \leqq \frac{d(f Q)}{d(f R)} \leqq M
$$

whenever $Q, R \in \mathscr{H}(D)$ with $Q \cap R \neq \emptyset$. We may assume that $Q \neq R$. By symmetry, it suffices to prove the second inequality. Since $Q(5) \subset D$, it follows from [V $\ddot{a}_{4}$, 2.4 ] that $f \mid \bar{Q}(3)$ is $\eta$-quasisymmetric with some $\eta$ depending only on $K$ and $n$. Let $z \in Q$. Then $\left|z-z_{Q}\right| \leqq\left|z_{R}-z_{Q}\right| \sqrt{n}$. Since $z_{R} \in \bar{Q}(3)$, this implies $\left|f(z)-f\left(z_{Q}\right)\right| \leqq$ $\eta(\sqrt{n})\left|f\left(z_{R}\right)-f\left(z_{Q}\right)\right|$, and hence

$$
d(f Q) \leqq 2 \eta(\sqrt{n})\left|f\left(z_{R}\right)-f\left(z_{Q}\right)\right| .
$$

Let $y$ be a vertex of $R$. Then $\left|z_{Q}-z_{R}\right| \leqq 3\left|y-z_{R}\right|$, which implies

$$
\left|f\left(z_{Q}\right)-f\left(z_{R}\right)\right| \leqq \eta(3) d(f R),
$$

and we obtain (7.19) with $M=2 \eta(\sqrt{n}) \eta(3)$.

We let $\alpha_{Q}$ be as in 6.13 but replace $\beta_{Q}^{f}$ by the affine map

$$
\gamma_{Q}^{f}(z)=\frac{z-f\left(z_{Q}\right)}{d(f Q)},
$$

and set $f_{Q}=\gamma_{Q}^{f} f \alpha_{Q}$. Let $\mathscr{G}_{K}$ be the family of all $K$-QC embeddings $g: I^{n}(2) \rightarrow R^{n}$ such that $g(0)=0$ and $d\left(g I^{n}\right)=1$. The compactness properties of QC maps imply that $\mathscr{G}_{K}$ is solid. Let $\mathscr{F}_{K}=\left\{g \mid I^{n}(3 / 2): g \in \mathscr{G}_{K}\right\}$. Using the notation of 7.7 we apply Lemma 3.9 and obtain the following variation of Lemma 7.8: 
7.20. Lemma. Let $\varepsilon^{\prime}>0, K \geqq 1$ and $\bar{K} \geqq 1$. Then there are $\delta=\delta\left(\varepsilon^{\prime}, K, n\right)>0$ and $K^{\prime}=K^{\prime}\left(\varepsilon^{\prime}, K, n, \bar{K}\right) \geqq \bar{K}$ with the following properties:

Let $Q \in \mathscr{H}(D)$, let $h: V_{Q}(D, 3 / 2) \rightarrow R^{n}$ be a $\bar{K} \cdot \mathrm{QC}$ LIP embedding, and let $g \in \mathscr{F}_{K}$ such that $d\left(g, h ; V_{Q}(D, 3 / 2)\right) \leqq \delta$. Then there is a LIP embedding $h^{\prime}: I^{n}(3 / 2) \rightarrow R^{n}$ such that

(1) $d\left(h^{\prime}, g ; I^{n}(4 / 3)\right) \leqq \varepsilon^{\prime}$,

(2) $h^{\prime}=h$ in $W_{Q}(4 / 3)$,

(3) $h^{\prime} \mid I^{n}(4 / 3)$ is $K^{\prime}$-QC.

We introduce the numbers $q$ and $\delta_{N}, \ldots, \delta_{0}$ as in 7.9, where now $\delta_{N}=$ $\min (1, q /(M+2))$ and $M$ is the constant of (7.19). Moreover, we set $K_{r}=1$ and $K_{j}=K^{\prime}\left(\delta_{j}, K, n, K_{j-1}\right)$ for $1 \leqq j \leqq N$. Corresponding to Lemma $7 \cdot 10_{j}$ we prove for every $j \in[0, N]$ :

$7.21_{j}$. Lemma. There is a LIP embedding $F_{j}: V_{j}(D) \rightarrow D^{\prime}$ with the following properties:

(1) $d\left(F_{j}, f ; Q\left(1+2^{-j-1}\right)\right) \leqq \delta_{j} d(f Q)$ for every $Q \in \mathscr{H}_{j}^{*}(D)$.

(2) $F_{j} Q\left(1+2^{-j-1}\right) \subset f Q(3 / 2)$ for every $Q \in \mathscr{H}_{j}^{*}(D)$.

(3) $F_{j}$ is $K_{j}$-QC.

Proof. Assume that $7.21_{j-1}$ is true. Define $F_{j}(x)=F_{j-1}(x)$ for $x \in W_{j-1}(D)^{\circ}$ Let $Q \in \mathscr{H}_{j}(D)$ and set $h_{Q}=\gamma_{Q}^{f} F_{j-1} \alpha_{Q} \mid V_{Q}(D, 3 / 2)$. By (7.19) we obtain

$$
d\left(h_{Q}, f_{Q} ; V_{Q}(D, 3 / 2)\right) \leqq \delta\left(\delta_{j}, K, n\right) .
$$

Hence we can apply 7.20 with the substitution $\varepsilon^{\prime} \mapsto \delta_{j}, K \mapsto K, \bar{K} \mapsto K_{j-1}, h \mapsto h_{Q}$, $g \mapsto f_{Q} \mid I^{n}(3 / 2)$. We obtain a LIP embedding $h_{Q}^{\prime}: I^{n}(3 / 2) \rightarrow R^{n}$ and define $F_{j}=$ $\left(\gamma_{Q}^{f}\right)^{-1} h_{Q}^{\prime} \alpha_{Q}^{-1}$ in $Q\left(1+2^{-j-1}\right)$. As in 7.10 , we can verify that $F_{j}: V_{j}(D) \rightarrow D^{\prime}$ satisfies the conditions (1), (2), (3) and is a LIP embedding.

The proof of Theorem 7.18 can now be completed as in 7.11. The desired map $F$ is $F_{N}$, which is $K_{N} \cdot \mathrm{QC}$ with $K_{N}$ depending only on $K$ and $n$. Indeed, if $x \in Q \in \mathscr{H}(D)$, it follows from the definition of $\mathscr{H}(D)$ that

$$
\left|F_{N}(x)-f(x)\right| \leqq \delta_{N} d(f Q) \leqq d(f Q) \leqq \varepsilon(x) .
$$

\section{References}

[Ah] AhLfors, L. V.: Lectures on quasiconformal mappings. - Van Nostrand Mathematical Studies 10, D. Van Nostrand Company, Inc., Princeton, N. J.-Toronto-New York, N. Y.-London, 1966.

[BG] Brown, M., and H. Gluck: Stable structures on manifolds I. - Ann. of Math. 79, 1964, $1-17$.

[EK] Edwards, R. D., and R. C. KIRBY: Deformations of spaces of imbeddings. - Ann. of Math. 93, 1971, 63-88. 
-Fe] Federer, H.: Geometric measure theory. - Springer-Verlag, Berlin-Heidelberg-New York, N. Y., 1969.

[GVs] GAULD, D. B., and J. VÄISÄLÄ: Lipschitz and quasiconformal flattening of spheres and cells. - Ann. Acad. Sci. Fenn. Ser. A I Math. 4, 1979, 371-382.

[GVm $]_{1}$ Gauld, D. B., and M. K. Vamanamurthy: Quasiconformal extensions of mappings in n-space. - Ann. Acad. Sci. Fenn. Ser. A I Math. 3, 1977, 229-246.

[GVm $\left.{ }_{2}\right]$ Gauld, D. B., and M. K. Vamanamurthy: A special case of Schönflies theorem for quasiconformal mappings in $n$-space. - Ann. Acad. Sci. Fenn. Ser. A I Math. 3, 1977, $311-316$.

[Ge] Gehring, F. W.: The Carathéodory convergence theorem for quasiconformal mappings in space. - Ann. Acad. Sci. Fenn. Ser A I Math. 336/11, 1964, 1-21.

[GO] Gehring, F. W., and B. G. OsGood: Uniform domains and the quasihyperbolic metric. J. Analyse Math. 36, 1979, 50-74.

[GP] Gehring, F. W., and B. P. Palka: Quasiconformally homogeneous domains. - J. Analyse Math. 30, 1976, 172-199.

[Jo] John, F.: On quasi-isometric mappings I. - Comm. Pure Appl. Math. 21, 1968, 77-110.

[Kk] KIIKKA, M.: Piecewise linear approximation of quasiconformal and Lipschitz homeomorphisms. - Ann. Acad. Sci. Fenn. Ser. A I Math. Dissertationes 30, 1980, 1-24.

[Kr] KIRBY, R. C.: Lectures on triangulations of manifolds. - Mimeographed notes, UCLA, 1969.

[LeV ${ }_{1}$ Lehto, O., and K. I. Virtanen: On the existence of quasiconformal mappings with prescribed complex dilatation. - Ann. Acad. Sci. Fenn. Ser. A I Math. 274, 1960, $1-24$.

[ $\left.\mathrm{LeV}_{2}\right]$ Lehto, O., and K. I. VIRTANen: Quasiconformal mappings in the plane. - Springer-Verlag, Berlin-Heidelberg-New York, N. Y., 1973.

[LT] LuUkKainen, J., and P. Tukia: Quasisymmetric and Lipschitz approximation of embeddings. - Ann. Acad. Sci. Fenn. Ser. A I Math. 6, 1981, 343-367.

[LuV] LuukKainen, J., and J. VÄIsÄLÄ: Elements of Lipschitz topology. - Ann. Acad. Sci. Fenn. Ser. A I Math. 3, 1977, 85--122.

[Mu] Munkres, J.: Elementary differential topology. - Annals of Mathematics Studies, No. 54, Princeton University Press, Princeton, N. J., 1963.

[Nä] NäÄTÄNEN, M.: Dilatation estimates for quasiconformal extensions. - Israel J. Math. 29, $1978,346-356$.

[RR] Rado, T., and P. V. Reichelderfer: Continuous transformations in analysis. - SpringerVerlag, Berlin-Göttingen-Heidelberg, 1955.

[RS] Rourke, C. P., and B. J. SANDERson: Introduction to piecewise-linear topology. - Springer-Verlag, Berlin-Heidelberg-New York, N. Y., 1972.

[St] SteIN, E. M.: Singular integrals and differentiability properties of functions. - Princeton University Press, Princeton, N. J., 1970.

[Su] Sullivan, D.: Hyperbolic geometry and homeomorphisms. - Geometric topology (Proceedings Georgia Topology Conf., Athens, Ga., 1977) edited by J. C. Cantrell, Academic Press, New York, N. Y.-London, 1979, 543-555.

[Th] Thurston, W. P.: The geometry and topology of three-manifolds. - Mimeographed notes, Princeton University, 1980.

[T $\left.\mathrm{Tu}_{1}\right]$ TukıA, P.: Lipschitz approximation of homeomorphisms. - Ann. Acad. Sci. Fenn. Ser. A I Math. 4, 1978/79, 137-144.

[ $\mathrm{Tu}_{2}$ ] TukiA, P.: A quasiconformal group not isomorphic to a Möbius group. - Ann. Acad. Sci. Fenn. Ser. A I Math. 6, 1981, 149-160.

[TV $\left.{ }_{1}\right]$ TukIA, P., and J. VÄIsÄLÄ: Quasisymmetric embeddings of metric spaces. - Ann. Acad. Sci. Fenn. Ser. A I Math. 5, 1980, 97-114. 
[ $\left.\mathrm{TV}_{2}\right]$ TUKIA, P., and J. VÄIsÄLä: Quasiconformal extension from dimension $n$ to $n+1$. - Ann. of Math., to appear.

[Vä] VÄIsÄLÄ, J.: Lectures on $n$-dimensional quasiconformal mappings. - Lecture Notes in Mathematics 229, Springer-Verlag, Berlin-Heidelberg-New York, N. Y., 1971.

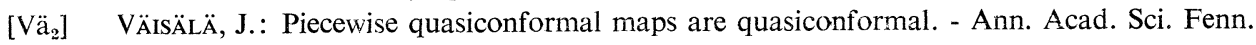
Ser. A I Math. 1, 1975, 3-6.

[V $\ddot{a}_{3}$ ] VÄıs̈̈LÄ, J.: Piecewise linear approximation of lipeomorphisms. - Ann. Acad. Sci. Fenn. Ser. A I Math. 3, 1977, 377-383.

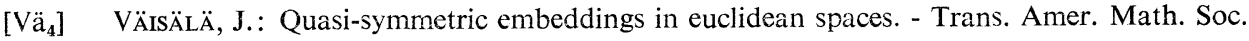
264, 1981, 191-204.

[Vu] Vuorinen, M.: Capacity densities and angular limits of quasiregular mappings. - Trans. Amer. Math. Soc. 263, 1981, 343-354.

[Wh] Whitney, H.: Analytic extensions of differentiable functions defined in closed sets. - Trans. Amer. Math. Soc. 36, 1934, 63-89.

University of Helsinki

Department of Mathematics

SF-00100 Helsinki 10

Raceived 7 May 1981 